\title{
A Numerical Study on the Improvement of Suction Performance and Hydraulic Efficiency for a Mixed-Flow Pump Impeller
}

\author{
Sung Kim, Kyoung-Yong Lee, Jin-Hyuk Kim, and Young-Seok Choi \\ Thermal \& Fluid System RઐBD Group, Korea Institute of Industrial Technology, 89 Yangdaegiro-gil, Ipjang-myeon, Seobuk-gu, \\ Cheonan-si, Chungcheongnam-do 331-822, Republic of Korea \\ Correspondence should be addressed to Jin-Hyuk Kim; jinhyuk@kitech.re.kr
}

Received 28 April 2014; Revised 11 September 2014; Accepted 16 September 2014; Published 8 October 2014

Academic Editor: Shaofan Li

Copyright (C) 2014 Sung Kim et al. This is an open access article distributed under the Creative Commons Attribution License, which permits unrestricted use, distribution, and reproduction in any medium, provided the original work is properly cited.

\begin{abstract}
This paper describes a numerical study on the improvement of suction performance and hydraulic efficiency of a mixed-flow pump by impellers. The design of these impellers was optimized using a commercial CFD (computational fluid dynamics) code and DOE (design of experiments). The design variables of meridional plane and vane plane development were defined for impeller design. In DOE, variables of inlet part were selected as main design variables in meridional plane, and incidence angle was selected in vane plane development. The verification of the experiment sets that were generated by $2^{k}$ factorial was done by numerical analysis. The objective functions were defined as the NPSHre (net positive suction head required), total efficiency, and total head of the impellers. The importance of the geometric design variables was analyzed using $2^{k}$ factorial designs. The interaction between the NPSHre and total efficiency, according to the meridional plane and incidence angle, was discussed by analyzing the $2^{k}$ factorial design results. The performance of optimally designed model was verified by experiments and numerical analysis and the reliability of the model was retained by comparison of numerical analysis and comparative analysis with the reference model.
\end{abstract}

\section{Introduction}

The mixed-flow pump is a typical instrument of mass energy consumption although it also has important roles in water resource development and various industrial plants. The importance of design engineering of the mixed-flow pump with high total efficiency and suction performance and energy saving as a consequence is increasing as oil price increases. The advanced enterprises in developed countries like the US, Germany, and Japan have competitiveness through their own technologies and know-how in pump designing that has been accumulated for more than a hundred years.

Specifically for the pumps that are used in higher value added business such as desalination or extraction of petroleum, the total efficiency and suction performance are a main issue giving advanced enterprises possessing accumulated technologies absolute advantage. For that reason, there have been researches going on regarding pumps including mixed-flow pump and the results have been reported several times by different companies and research agencies $[1,2]$.
When it comes to impeller designing of the mixed-flow pump, two main objectives of the research, namely, high total efficiency and improved suction performance, have conflicting directions of the design $[3,4]$. The design technique that could improve suction performance maintaining high total efficiency at the same time is very important but more design experiences are required at this stage.

Suction performance correlate with cavitation. In case where the flow level changes by time when the pump is operating, unusual phenomena such as cavitation happen as the pressure of pump inlet part changes. Because of the nature of the fluid, the suction performance of a pump decreases creating bubbles when the pressure is low. Thus, securing design technology for pump with high suction performance is very important [5-7].

In order to improve total efficiency and suction performance of the impeller by selecting impeller shape variables appropriately, an analysis that can determine the importance of the design variables and the effect of chosen variables on pump performance is required. In order to perform the study, numerical analysis and bench marking via experiments were 
done on the reference model and the results of them were used as criteria for the comparison.

Recently, the application of computational fluid dynamics (CFD) in the performance analysis of fluid machinery is common, and many studies have conducted for validating its usefulness in predicting the performance of pumps. For instance, Goto and Zangeneh [8] applied their optimization methodology based on an inverse design method and threedimensional numerical analysis to design low specific speed pump diffusers with high efficiency performance. Bing and Cao [9] also improved the total hydraulic efficiency of a mixed-flow pump impeller by a combined approach using numerical simulation, inverse design, and a genetic algorithm. J. H. Kim and K. Y. Kim [10] tried the single-objective numerical optimization to enhance the total efficiency of a mixed-flow pump with two design variables related to vaned diffuser geometry.

In design engineering, the importance of the mixedflow pump with high total efficiency and suction performance has been increasing, which has made it necessary to improve hydrodynamic design techniques in order to develop a mixed-flow pump with high total efficiency and optimal suction performance. Because the most important component of the pump is the impeller, its hydrodynamic design determines the pump design technique.

The two main objectives in designing the impeller of the mixed-flow pump, namely, high total efficiency and improved suction performance, can be realized using contrasting techniques. A design technique that could improve suction performance and maintain high total efficiency at the same time is desirable but remains in the experimental stage.

In this study, the design variables of the impeller meridional plane and vane plane development were defined for the improvement of suction performance. The design variables were then rated in importance by systematically analyzing them in relation to the response variables of the impeller. First, an impeller with high total efficiency, which satisfies the head at the design flow rate, was designed by using database $(D / B)$.

The impeller was designed using $D / B, 2^{k}$ factorial designs, which were applied to analyze the effect on suction performance and total efficiency of the design variables. The tendency to change performance according to each variation in shape was studied by numerical analysis. By applying response optimization, the main design variables determined by the $2^{k}$ factorial design were used to create the optimal shape.

The performance of optimized impeller was verified by numerical analysis and experiments. Numerical analysis and experiment of the reference model were used to compare the optimal impeller with the reference model.

\section{Mixed-Flow Pump Impeller Design Method}

2.1. Traditional Design Method. The traditional design for the meridional plane and the front plane of the impeller is done easily by applying pump theory. When design specifications are given, the diameters of the inlet/outlet of the meridional plane are determined by basic pump theory. The shroud and hub curve of the inlet/outlet of the meridional plane are easily connected by drawing an arc.

In designing the blade shape, the inlet/outlet angles of the blade are mainly determined by the flow angles of the inlet/outlet using the given meridional plane, according to pump design theory. The sweep angle, which is relevant to the blade length, is determined by the inlet/outlet angles because it is designed such that it connects the inlet/outlet angles smoothly when the angles are determined.

As shown in Figure 1, the shape of the impeller is presented in the meridional and front planes; Figure 1(a) shows the meridional view of the blade shape and presents information on the direction of axis and radius; Figure 1(b) shows the front plane representing the radius and rotational direction; and Figure 1(c) shows the vane plane development of the impeller. The vane plane development includes the blade angle distribution. Axis $x$ of the vane plane development indicates the total length of the arc at each radius from the front view. Axis $y$ indicates the total length of the blade in the meridional view. Hence, the distribution of the blade angles is easily seen in the vane plane development and is usually achieved by the smooth connection of the inlet blade angle and the outlet blade angle. The inlet and outlet angles of the blade are mainly derived using pump design theory $[11,12]$.

The head can be satisfied by a traditionally designed impeller but, in order to improve total efficiency and suction performance, a detailed definition of the design variables and subsequent analysis of performance variations according to different impeller design variables are required.

The meridional plane shows the shape and size of blade. The impeller meridional shape was designed using our own $D / B$. Figure 2 shows the newly designed impeller shape. As shown in Figure 2, consequentially, the axis direction of the newly designed shape is approximate $24 \%$ shorter than that of the reference model.

2.2. Design Variables in a Meridional Plane. The typical shape of the meridional plane is shown in Figure 1(a), and the design variables for this meridional plane are defined in Figure 3. On the inlet of the impeller, $R_{1} h$ is the hub, $R_{1} s$ is the inlet radius of shroud, and $\Phi_{1}$ indicates the inclined angle of the impeller's leading edge. On the outlet of the impeller, $R_{2}$ is the outlet radius, $B_{2}$ is the blade width of the impeller's trailing edge, and $\Phi_{2}$ indicates the inclined angle of the trailing edge. The outlet hub of the impeller and the straight line of the shroud are defined as $\% L \_h$ and $\% L_{-} s$, respectively. Because it can express smooth curves, a Bezier curve was used to connect the inlet to the outlet of the impeller. When using Bezier curves, two reference points with set inclination levels are connected smoothly using a control point, and the shape of the curve varies depending on the location of the control point. When the control point is determined, the curve is connected smoothly in accordance with the inlet/outlet angles. When the impeller hub curve meets the horizontal and vertical lines, $\theta_{1}-h$ and $\theta_{2}-h$ indicate the inlet angle and outlet angle, respectively. $\theta_{1} s$ and $\theta_{2} s$ 


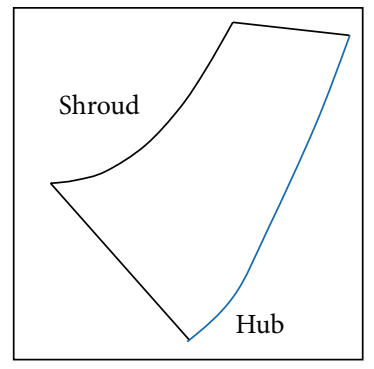

(a) Meridional plane

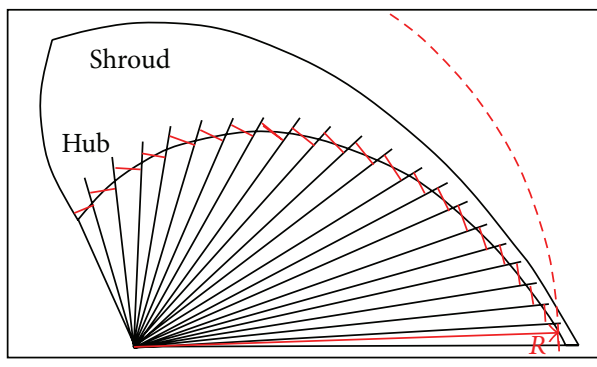

(b) Front plane

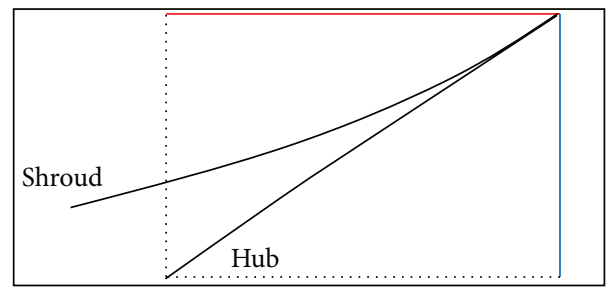

(c) Vane plane development

FIgure 1: Traditional impeller design method.

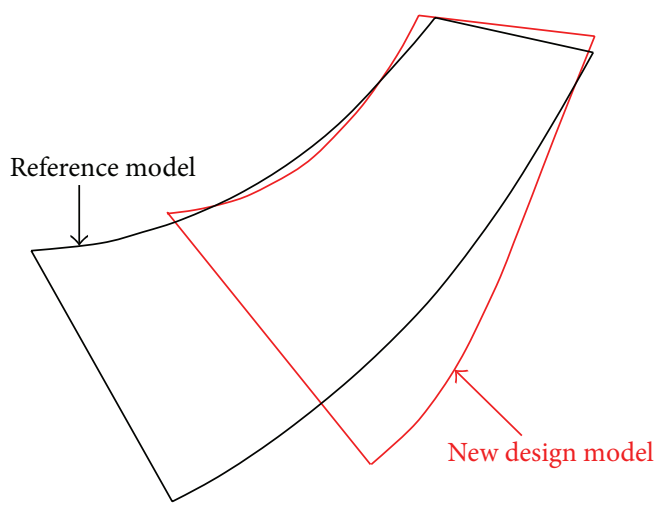

FIGURE 2: Comparison of meridional planes.

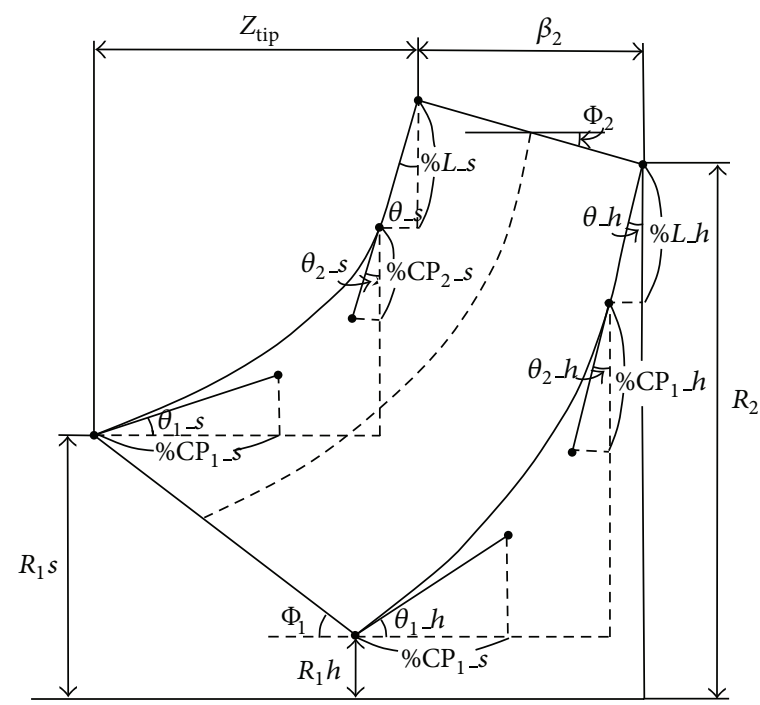

FIGURE 3: Design variable of meridional plane [11, 12]. 


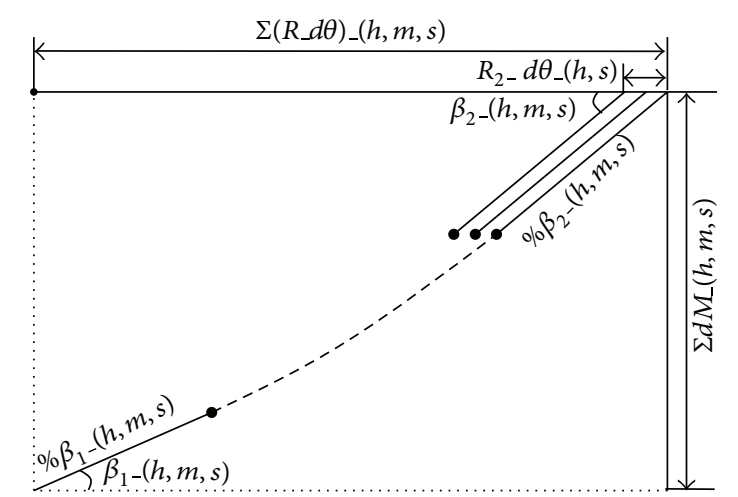

Figure 4: Design variable of vane plane development $[13,14]$.

indicate those of the shroud. $\theta h$ and $\theta_{s} s$ indicate the angles of the outlet hub and the shroud, respectively, when $\% L \_h$ and $\% L_{-} s$ of the straight lines meet the vertical axis. The control points of the hub inlet and outlet are shown as $\% \mathrm{CP}_{1}-h$ and $\% \mathrm{CP}_{2}-h$, respectively, and $\% \mathrm{CP}_{1}-s$ and $\% \mathrm{CP}_{2} s$ indicate those of the shroud. $Z_{\text {tip }}$ indicates the length of the axial direction from inlet/outlet parts of the shroud [11, 12].

2.3. Design Variables in a Vane Plane Development. A typical shape of vane plane development is shown in Figure 1(c), and the design variables for this vane plane development are defined in Figure 4 . In the diagram, " $h$ " means hub, " $m$ " means midspan, and " $s$ " means shroud. $\Sigma\left(R_{-} d \theta\right)_{-}(h, m, s)$ indicates the total length of the arc at each radius from the front view. $\Sigma d M_{-}(h, m, s)$ represents the total values of blade length in the meridional view; $\% \beta_{1-}(h, m, s)$ and $\% \beta_{2}-(h, m, s)$ show the portion of the blade having the same blade angle at the leading edge and trailing edge, respectively, and they are presented as a percentage of the whole length of the $y$-axis. $\beta_{1-}(h, m, s)$ is the inlet angle of the blade from the impeller, and $\beta_{2}-(h, m, s)$ is the outlet angle of the blade from the impeller. $R_{2} d \theta_{-}(h, s)$ shows the inclination level toward the circumference from the hub and shroud at the outlet of the impeller. The inlet and outlet sections are connected by a smooth curved line, the angle of which changed linearly. Figure 5 shows the three-dimensional geometry of the impeller using the variables of the meridional plane and the vane plane development $[13,14]$.

\section{Numerical Analysis Methods}

The three-dimensional shape of the impeller was generated using the ANSYS CFX-BladeGen program. The structured grid system was generated using ANSYS CFX-TurboGrid, a program that generates a fluid machinery grid [15].

Although the impeller has five blades, we carried out the numerical analysis on only one blade passage using a periodic condition. Figure 6 shows the boundary conditions for the impeller calculation. Water with ambient temperature of $25^{\circ} \mathrm{C}$ was specified as the working fluid. We set the atmospheric pressure of $1 \mathrm{~atm}$ with the turbulence intensity of $5 \%$ on the inlet section of the impeller and gave the mass flow rate of

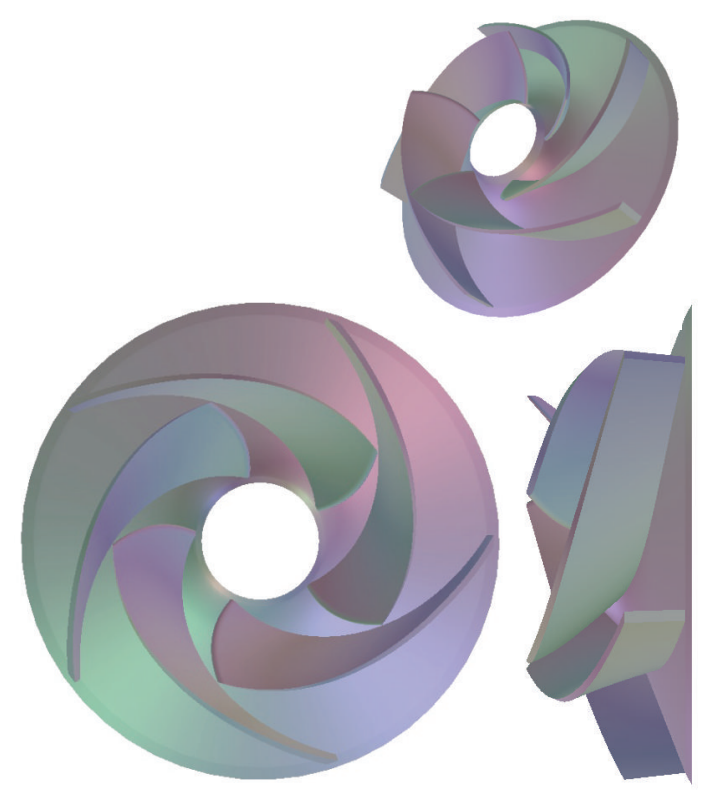

FIgURE 5: Three-dimensional geometry of the impeller.

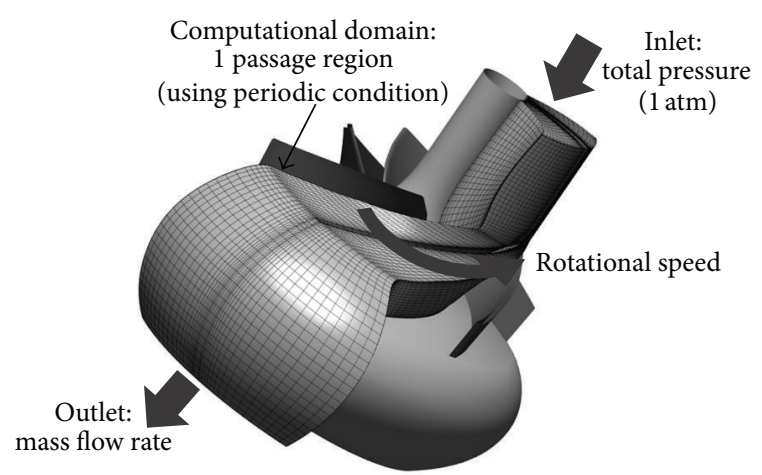

FIGURE 6: Boundary conditions for the impeller calculation.

$665.33 \mathrm{~kg} / \mathrm{s}$ of one blade passage at the design point on the exit section as a boundary condition. The solid surfaces in the computational domain were considered to be hydraulically smooth with adiabatic and no-slip conditions. The rotational speed of the impeller was $580 \mathrm{r} / \mathrm{min}$. When the numerical analysis was performed for the impeller only, the inlet was simplified as a straight pipe and the outlet was expressed as the same meridional shape without the diffuser vane.

A structured grid system was constructed in the computational domain, with O-type grids near the main blade and vane surfaces and $\mathrm{H}$-type grids in the other regions. Figure 7 shows the results of the grid dependency test for the total head and total efficiency of the reference impeller model. The total head and total efficiency values of the reference impeller did not change as the grid size was varied from approximately 90,000 to 110,000 . Thus, about 90,000 grid points were used to define the computational domain encompassing the main 


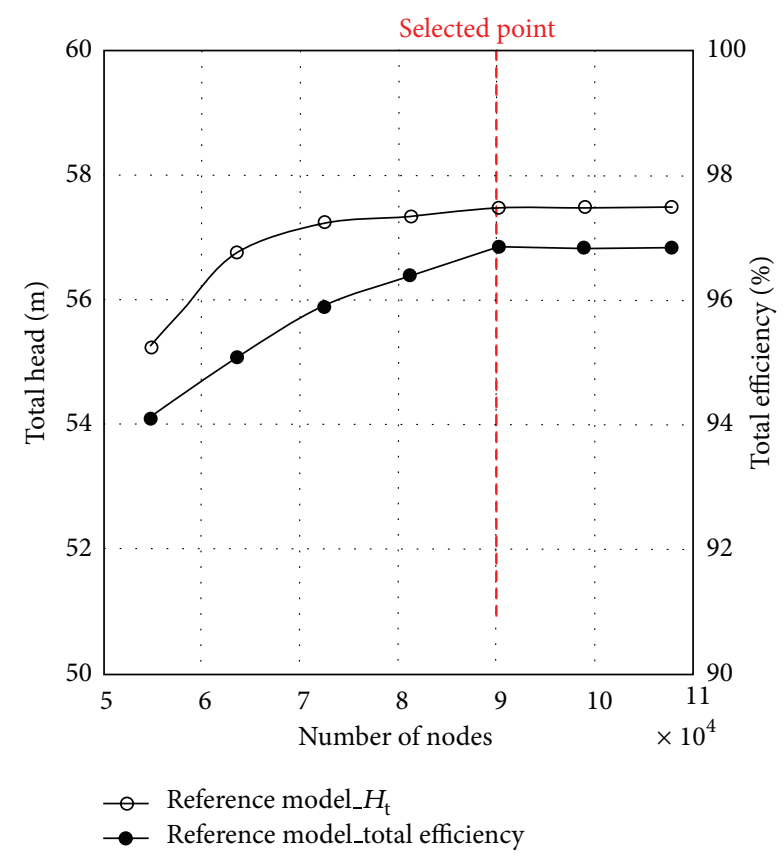

FIgURE 7: Results of the grid dependency test.

impeller passage. Figure 8 shows a typical example of the grid system used for the mixed-flow pump impeller in this work.

The ANSYS CFX-11, which is a commercial computational fluid dynamics (CFD) code, was used for the numerical analysis. A three-dimensional Reynolds average Navier-Stokes equation was used to analyze incompressible turbulence flow inside the pump. The governing equation was discretized using a finite volume method. A high-resolution scheme, which has more than a second degree of accuracy, was used to solve the convection-diffusion equations. For the turbulent model, the shear stress transport $k-\omega$ model [1619], which is appropriate for the prediction of flow separation, was used to analyze turbulent flow through the impeller.

We used water as a working fluid. Disk friction losses, mechanical losses, leakage losses, and the tip clearance effect were not included in this calculation. The specific speed $\left(\mathrm{r} / \mathrm{min}, \mathrm{m}^{3} / \mathrm{min}, \mathrm{m}\right)$ of the pump was 380 , the flow rate was $12,000 \mathrm{~m}^{3} / \mathrm{hr}$, and the total head was $60 \mathrm{~m}$.

\section{Design of Experiments}

The experiments design was based on the modern analysis of statistics, which helps select the main cause of abnormal fluctuations from many possible causes. In this study, $2^{k}$ factorial designs of the design of experiment (DOE) were used as numerical optimization methods [21-23]. Minitab 14, a commercial program, was used for the analysis of DOE.

Figure 9 shows the flow chart of this study. The performance of the reference model with same specific speed was analyzed and drawbacks that reduce pump performance could be identified based on the result of the analysis. Impeller design variables for the improvement of pump performance were defined using $D / B$. Response variables

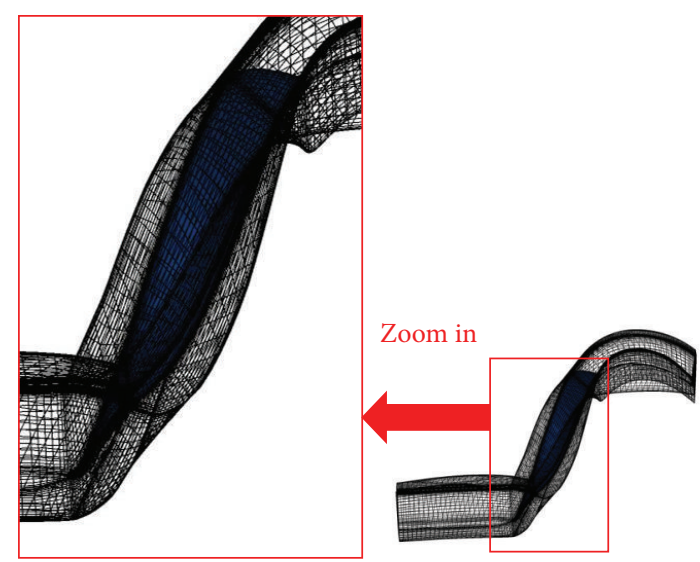

FIgURE 8: Grid system for numerical analysis of the impeller.

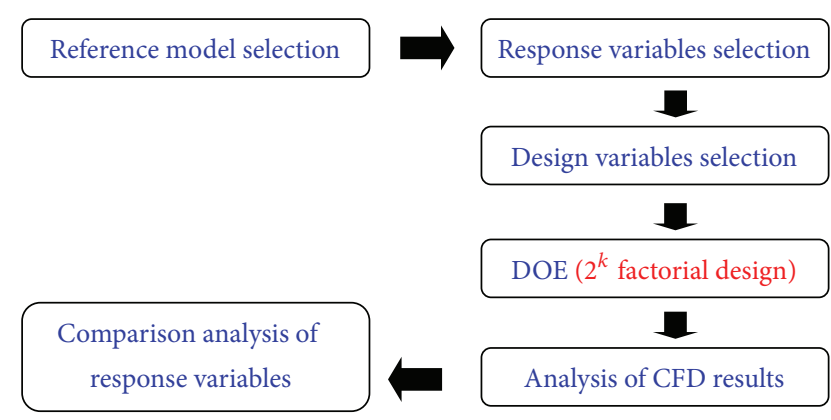

FIgURE 9: Optimization process flowchart.

were defined as total head, total efficiency, and suction performance. Tendencies of the response variables according to the identified design variables were analyzed by using $2^{k}$ factorial. The response variables of experiment sets generated by $2^{k}$ factorial were verified by using numerical analysis. Impeller shape was deduced by response optimization with the result of $2^{k}$ factorial. The performance of the optimized model was compared to that of the reference model using comparative analysis.

In DOE, a response variable should be defined in order to analyze the performance of the impeller according to the design variables. The actual response variables are defined in the total head curve, the total efficiency curve, and the NPSH curve, as shown in Figures 10 and 11. The design flow-rate is considered ideal if the flow rate at maximum total efficiency corresponds to the required flow rate.

In the case where the flow level changes over time when the pump is operating, unusual phenomena, such as cavitation, happen as the pressure of the pump inlet changes. Because of the nature of the fluid, the suction performance of the pump decreases, creating bubbles when the pressure is low. Thus, securing a design technology for a pump with high suction performance is very important. The graph of the results shows that net positive suction head (NPSH) value, which is represented as the $x$-axis, is determined by the set 
TABLE 1: Design target (real), design specifications, and CFD results of the reference model.

\begin{tabular}{lccc}
\hline & Design target (real) & Design specifications & CFD \\
\hline$Q\left(\mathrm{~m}^{3} / \mathrm{hr}\right)$ & 12,000 & 12,000 & 12,000 \\
Total head $(\mathrm{m})$ & 60 & More than $63 \mathrm{~m}$ & 57.48 \\
Total efficiency $(\%)$ & To be maximized & To be maximized & 96.84 \\
NPSHre $(\mathrm{m})$ & To be minimized & To be minimized & 6.7 \\
\hline
\end{tabular}

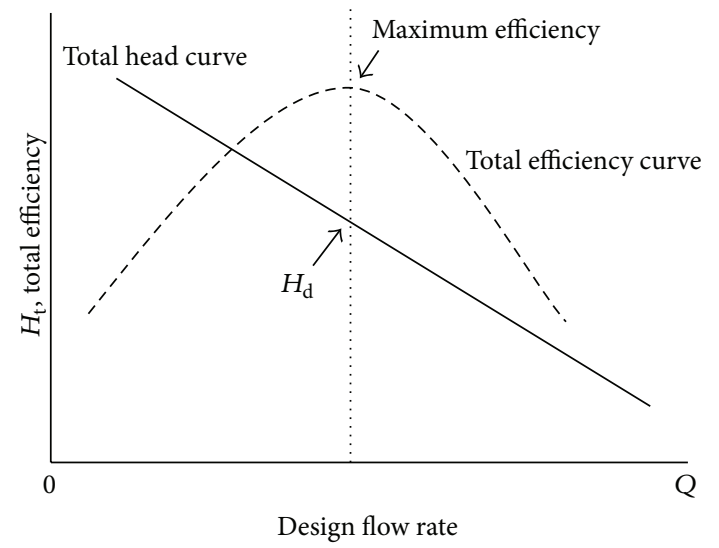

FIgURE 10: Total head and total efficiency curve.

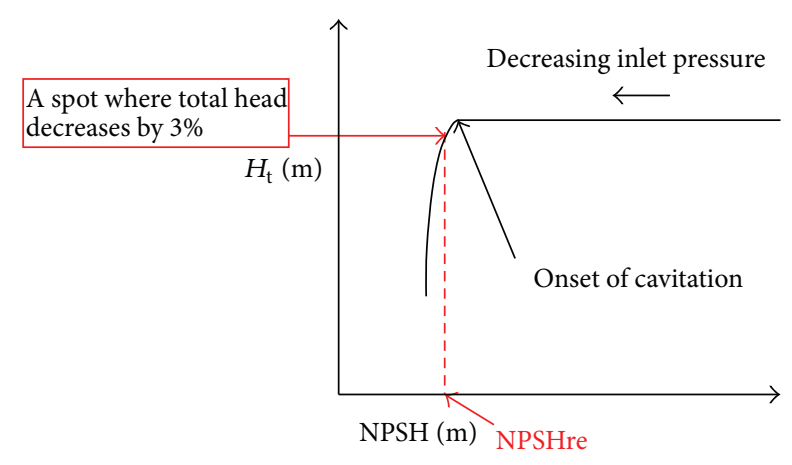

FIgURE 11: NPSH curve.

inlet pressure $\left(P_{\text {in }}\right)$ and saturation vapor pressure $\left(P_{\mathrm{v}}\right)$, and it is calculated by the following equation:

$$
\mathrm{NPSH}=\frac{P_{\text {in }}-P_{\mathrm{v}}}{\rho g} .
$$

Different NPSH values are generated as the inlet pressure decreases and NPSH required (NPSHre) represents an area with more than $3 \%$ of loss in the total head [24-26].

\section{Optimal Mixed-Flow Pump Impeller Design}

Table 1 shows the real design target and the modified design specifications for the CFD cases and the CFD results for the reference model. The specific speed $\left(\mathrm{r} / \mathrm{min}, \mathrm{m}^{3} / \mathrm{min}, \mathrm{m}\right)$ of the pump is 380 , the flow rate is $12,000 \mathrm{~m}^{3} / \mathrm{hr}$, the total head is $60 \mathrm{~m}$, the total efficiency should be maximized, and the NPSHre should be minimized at the design flow-rate. According to the numerical analysis of the reference model, however, the numerical analyses for the impeller do not satisfy $60 \mathrm{~m}$. As a result, a new design model that satisfies the specifications is needed.

Because of the simplified flow domain, without including tip clearance and roughness, the results of the numerical analysis are expected to be higher than those of the design target. Thus, we modified the design specifications for the CFD results. Design specifications for the numerical analysis are shown in Table 1. The total head was defined to be higher than $63 \mathrm{~m}$ from the impeller. The total efficiency should be maximized and the NPSHre should be minimized at the design flow rate.

5.1. $2^{k}$ Factorial Designs. $2^{k}$ factorial designs are usually represented as $n^{k}$. In this DOE, the number of factors is $k$ and the number of levels is $n$. The experiments are performed in every possible combination of all factors. The number of performed experiments should be at least $n^{k}$, without repetition. The advantage of factorial designs is that we can assume the main effect (the sole effect of the factor) and interaction effect (the effect between factors) of all factors. This convenient screening method can be used to find the core factor when there are many factors involved at the beginning of the experiment. In this study, considering the number of factors involved and the number of possible experiments, in addition to cost and time, we used fractional factorial designs in which the number of experiments is reduced by deleting less meaningful interactions.

5.2. Effect of Impeller Design Variables. To analyze the influence of the impeller design variables on the performance of the mixed-flow pump, the $2^{k}$ factorial designs were applied after defining the design variables of the meridional plane and the vane plane development. We therefore generated nine experimental conditions for the numerical analysis, including a center point using four design variables. As the various impeller design variables, the selected design variables for the $2^{k}$ factorial designs are $\mathrm{i} \beta_{1} h$ and $\mathrm{i} \beta_{1} s$, which are related to the incidence angle of the design variables of the vane plane development, and $R_{1} s$ and $Z_{\text {tip }}$, which are the meridional plane design variables. Here, the $\mathrm{i} \beta_{1} h$, and $\mathrm{i} \beta_{1} s$ are the incidence angles at the leading edge of the blade on the hub and shroud, respectively. The incidence angles are the design variables showing the difference between flow angle and the inlet angle. 

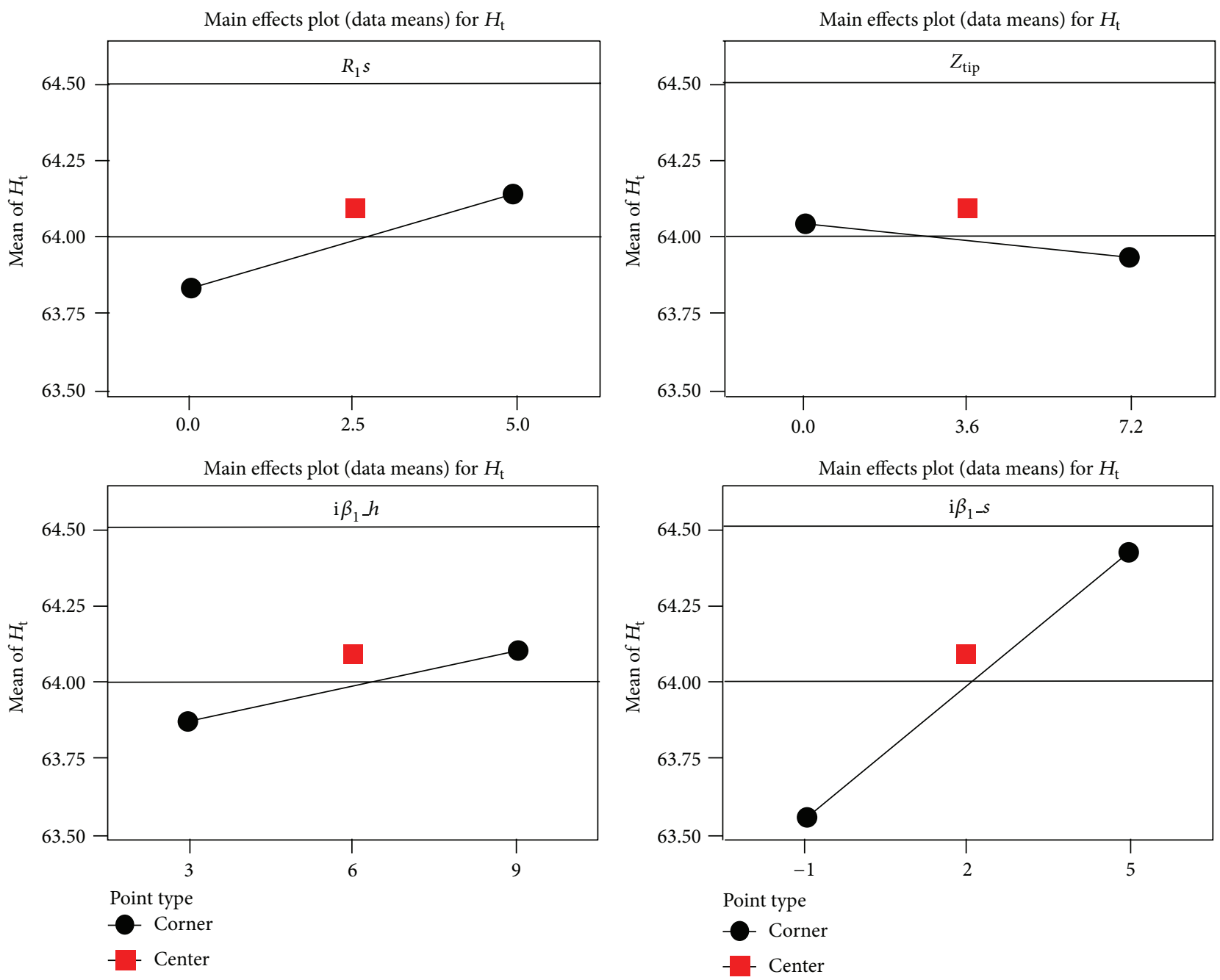

FIGURE 12: Main effects plot for total head.

TABLE 2: $2^{k}$ factorial designs set.

\begin{tabular}{lcccc}
\hline Set & $A$ & $B$ & $C$ & $\begin{array}{c}D \\
(A \times B \times C)\end{array}$ \\
\hline 1 & - & - & - & - \\
2 & + & - & - & + \\
3 & - & + & - & + \\
4 & + & + & - & - \\
5 & - & - & + & + \\
6 & + & - & + & - \\
7 & - & + & + & - \\
8 & + & + & + & + \\
Center & 0 & 0 & 0 & 0 \\
\hline
\end{tabular}

Table 2 shows the set arrangement of $2^{k}$ factorial designs for nine experimental conditions. Here, the symbols of "+" and "-" mean the maximum and minimum levels of each design variable, respectively. In Table 2 , the variables of $A, B$, and $C$ are defined by the orthogonal design and the $D$ variable is decided by the combination of $A \times B \times C$.

The base model applied in $2^{k}$ factorial designs was the shape used in a previous study, and it satisfied maximum total efficiency at the design flow rate. Variation ranges of $\mathrm{i} \beta_{1} h$ and $\mathrm{i} \beta_{1} s$ are $\pm 3^{\circ}$, and those of $R_{1} s$ and $Z_{\text {tip }}$ were $\pm 2.5 \%$ and $\pm 3.6 \%$, respectively. The rest of the design variables of the meridional plane and vane plane development were fixed as base design value. Table 3 shows the numerical analysis sets of $2^{k}$ factorial designs.

Figures 12 and 13 show plots of the main effects and a Pareto chart of the total head, respectively. In the main effects plot, the tendency to increase the total head can be identified as increases in $R_{1} s, \mathrm{i} \beta_{1} h$, and $\mathrm{i} \beta_{1} s$. In Pareto chart, the total head is influenced by i $\beta_{1} s, R_{1} s$, and i $\beta_{1} h$ in the order of most to least.

Figures 14 and 15 show plots of the main effects and Pareto chart of total efficiency, respectively. In the main effects plot, total efficiency tends to increase as $R_{1} s$ and $Z_{\text {tip }}$ decrease, but $\mathrm{i} \beta_{1} h$ and $\mathrm{i} \beta_{1_{-}} s$ increase. It is clear that the tendency of the meridional plane design variables is 


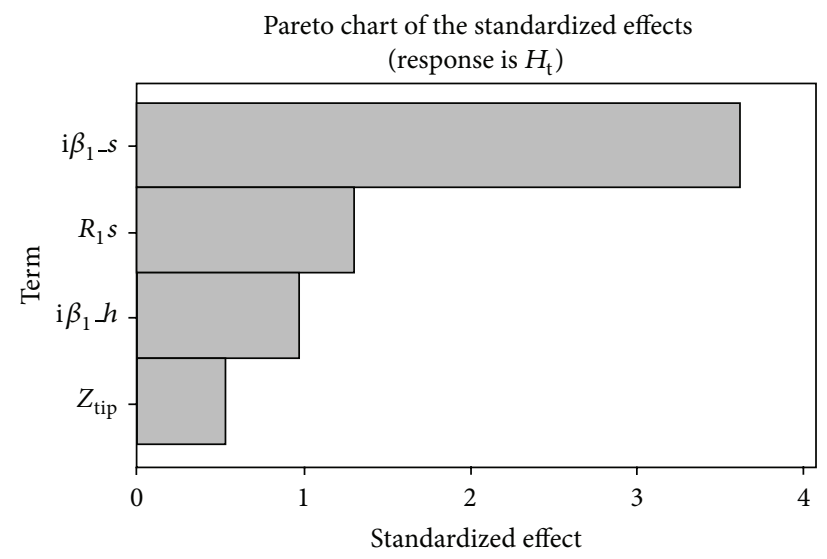

Figure 13: Pareto chart for total head.
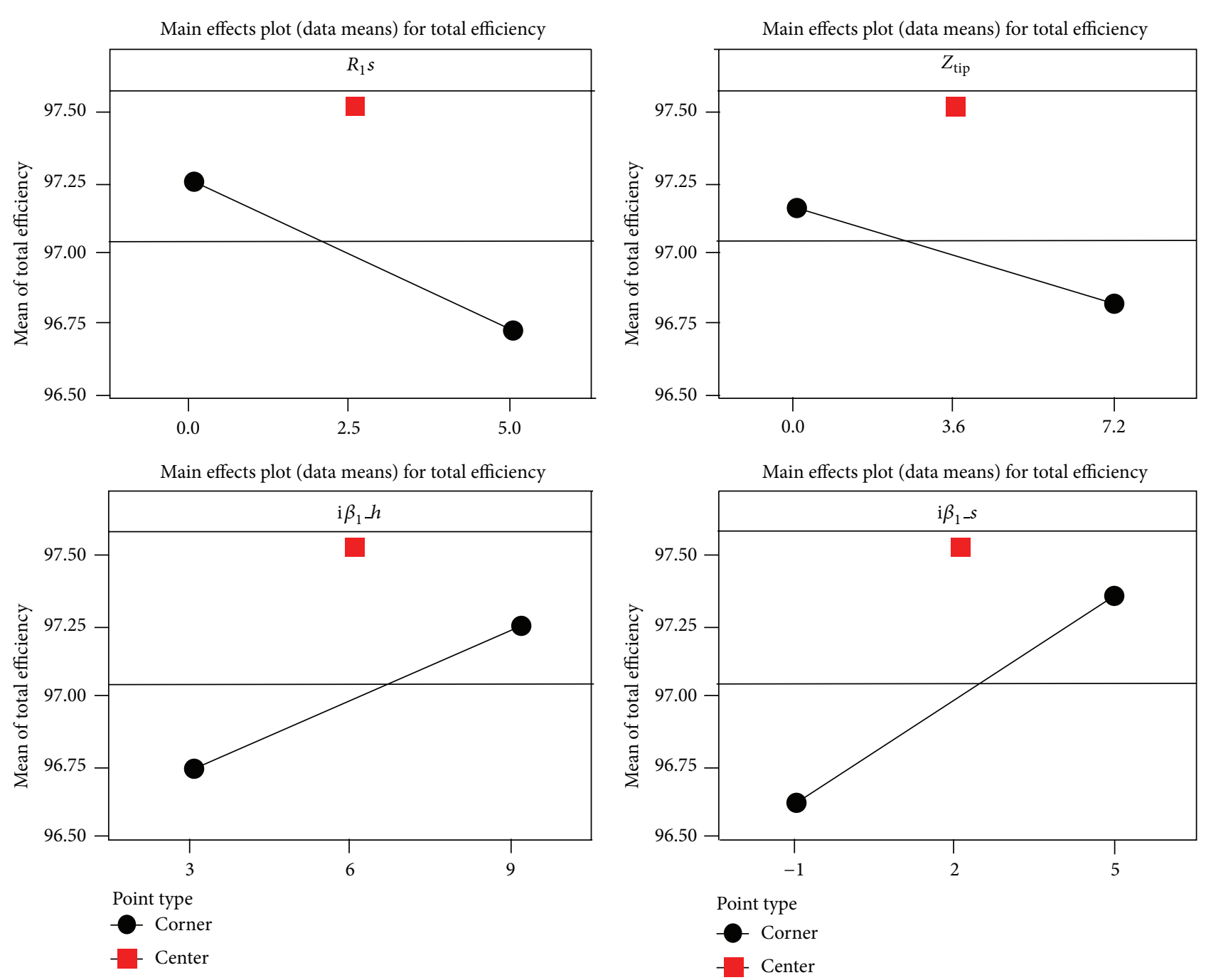

FIGURE 14: Main effects plot for total efficiency. 


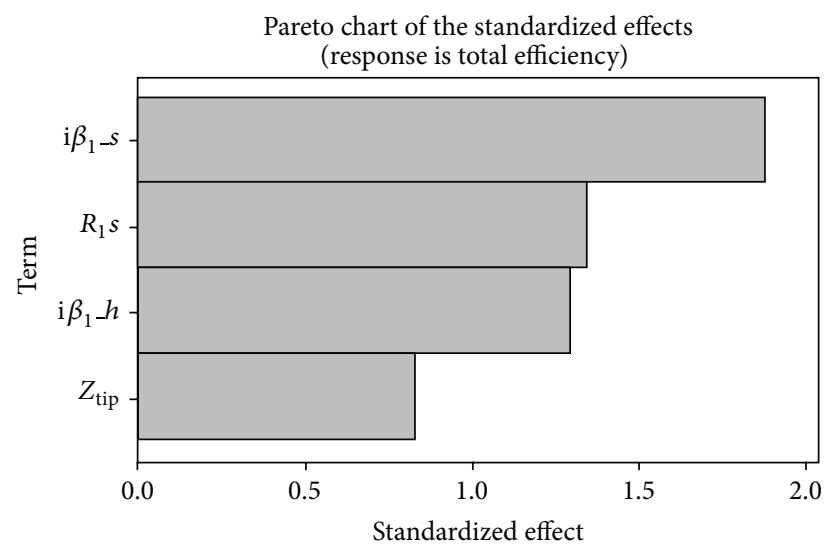

FIGURE 15: Pareto chart for total efficiency.

TABLE 3: Numerical analysis set of $2^{k}$ factorial designs for the impeller design.

\begin{tabular}{|c|c|c|c|c|c|c|c|}
\hline Set & $R_{1} s(\mathrm{~m})$ & $Z_{\text {tip }}(\mathrm{m})$ & $\mathrm{i} \beta\lrcorner h\left(^{\circ}\right)$ & $\mathrm{i} \beta \_h\left(^{\circ}\right)$ & Total head $(\mathrm{m})$ & Total efficiency (\%) & NPSHre (m) \\
\hline 1 & 0 & 0 & 3 & -1 & 63.24 & 96.65 & 6.36 \\
\hline 2 & 5 & 0 & 3 & 5 & 64.92 & 97.21 & 5.23 \\
\hline 3 & 0 & 7.2 & 3 & 5 & 64.07 & 97.61 & 5.64 \\
\hline 4 & 5 & 7.2 & 3 & -1 & 63.25 & 95.47 & 8.09 \\
\hline 5 & 0 & 0 & 9 & 5 & 64.18 & 97.55 & 6.15 \\
\hline 6 & 5 & 0 & 9 & -1 & 63.86 & 97.19 & 5.28 \\
\hline 7 & 0 & 7.2 & 9 & -1 & 63.83 & 97.18 & 5.74 \\
\hline 8 & 5 & 7.2 & 9 & 5 & 64.55 & 97.04 & 5.28 \\
\hline Center & 2.5 & 3.6 & 6 & 2 & 64.10 & 97.54 & 5.48 \\
\hline
\end{tabular}

opposite to that of the vane plane development. In the Pareto chart, i $\beta_{1} \_s, R_{1} s$, and $\mathrm{i} \beta_{1} \_h$ are variables affecting total efficiency; $\mathrm{i} \beta_{1} s$ shows the most influence and $\mathrm{i} \beta_{1} h$ shows the least.

Figures 16 and 17 show plots of the main effects and a Pareto chart of NPSHre. In the main effects plot, NPSHre tends to increase as $Z_{\text {tip }}$ decreases, but i $\beta_{1} h$ and $\mathrm{i} \beta_{1} s$ increase. Compared to other design variables, $R_{1} s$ does not have a significant effect on NPSHre. Apart from $R_{1} s$, it is clear that the tendency of the meridional plane design variables is opposite to that of the vane plane development. In the Pareto chart, i $\beta_{1}-s, \mathrm{i} \beta_{1}-h$, and $Z_{\text {tip }}$ are variables affecting total efficiency; i $\beta_{1-} s$ shows the most influence and $Z_{\text {tip }}$ shows the least.

To summarize the $2^{k}$ factorial designs, it was identified that the tendencies of total efficiency and NPSHre were opposite, depending on the design variables. The analysis of the tendencies of the response variables was important in order to draw a shape with improved NPSHre in high total efficiency, depending on the changing design variables. In order to create an impeller shape with improved NPSHre in high total efficiency, a response optimization method was applied. In Table 3 , most of the $2^{k}$ factorial sets satisfy $63 \mathrm{~m}$ of total head. Thus, the response variables were defined to improve total efficiency and NPSHre at the design flow rate, except for the total head as the objective function.

Response optimization was performed by regression analysis. Regression analysis is assuming mathematical model based on data in order to investigate the relevance among variables. Generally, the estimated model is used to make necessary prediction or statistical inference. Multiple regression analysis is a type of regression analysis where the relation between two or more predictors and one dependent variable is estimated linearly on straight line. Estimation equation for multiple regression analysis is as follows:

$$
\widehat{Y}=\widehat{\beta_{0}}+\widehat{\beta_{1}} \times X_{1}+\widehat{\beta_{2}} \times X_{2}+\cdots+\widehat{\beta_{n}} \times X_{n}
$$

where $X$ indicates the design variables from $2^{k}$ factorial. $\widehat{Y}$ and $\widehat{\beta}$ are established by regression analysis. The regression model with consideration of multiple regression models is as (3) and (4). 

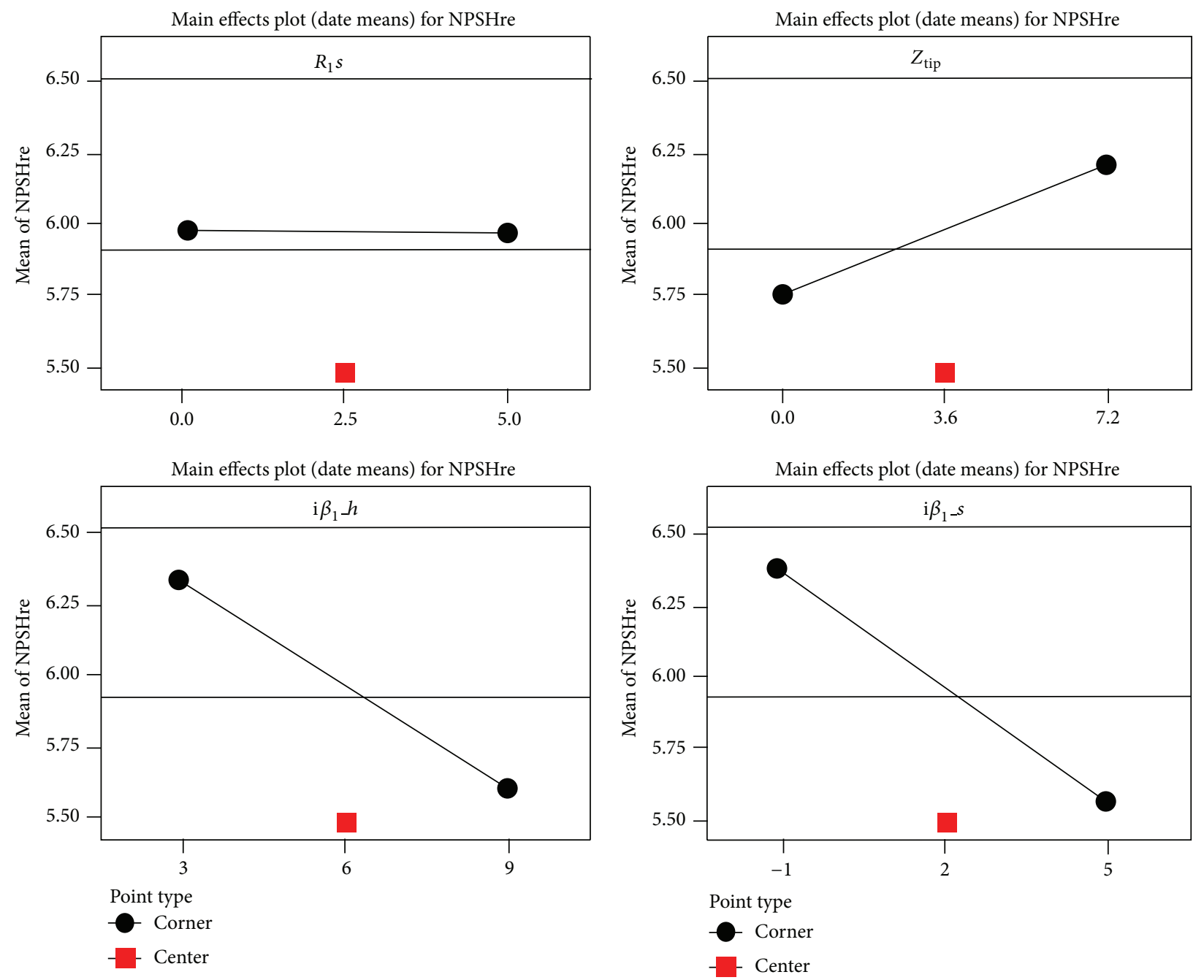

FIGURE 16: Main effects plot for NPSHre.

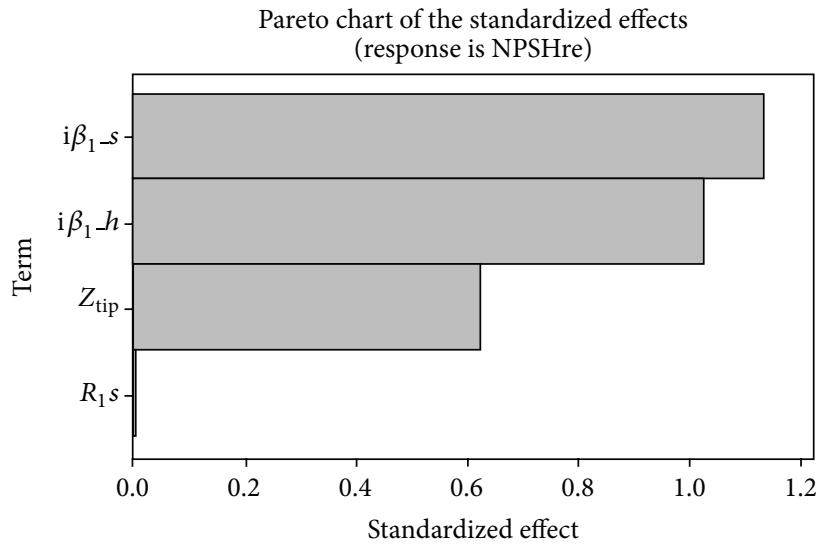

FIGURE 17: Pareto chart for NPSHre. 


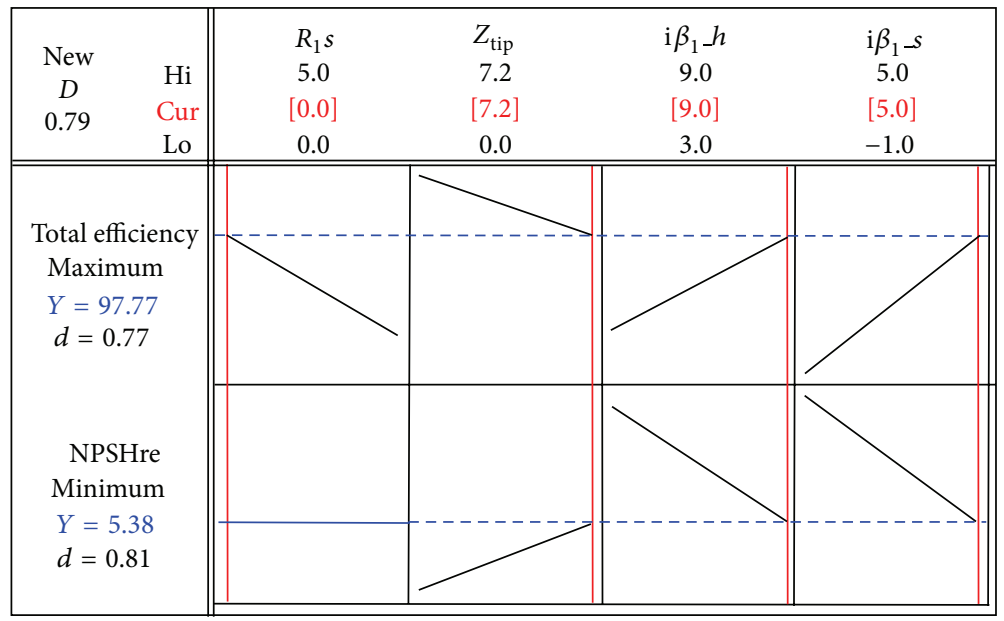

FIgURE 18: Plot for response optimization.

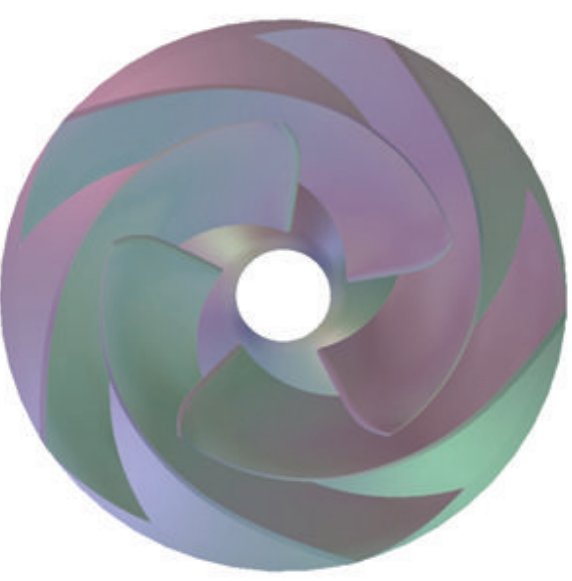

(a) Reference model

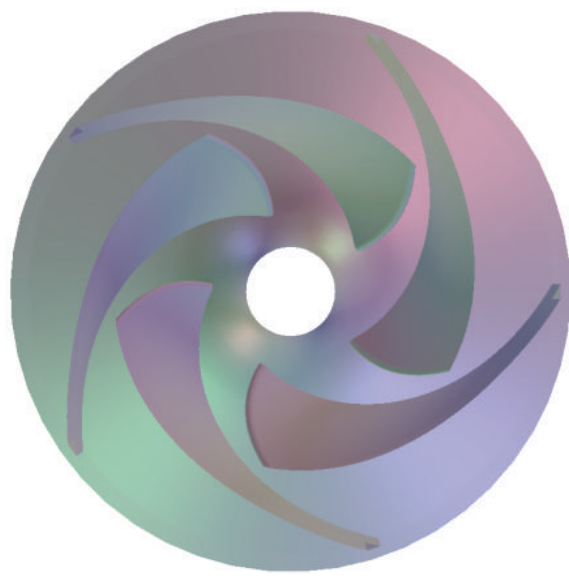

(b) Optimum model

FIGURE 19: Comparison of three-dimensional geometry impeller.

The regression equations for total efficiency and NPSHre are as follows, respectively:

$$
\begin{aligned}
\text { Total efficiency }= & 96.7-0.104 \times R_{1} s-0.0447 \times Z_{\text {tip }} \\
& +0.0839 \times \mathrm{i} \beta_{1} h+0.121 \times \mathrm{i} \beta_{1} s, \\
\text { NPSHre }= & 6.68-0.000 \times R_{1} s+0.0604 \times Z_{\text {tip }} \\
& -0.119 \times \mathrm{i} \beta_{1} h-0.132 \times \mathrm{i} \beta_{1} s .
\end{aligned}
$$

The regression models in (2) and (3) were produced considering each design variable and thereby applicable as reliable optimization and predictive models. In response optimization, the objective function was defined as maximum total efficiency and minimum NPSHre. The total head was excluded because it showed little change as the design variables changed. Figure 18 shows the plot of response optimization. As shown in Figure 18, the optimum model that satisfies the objective value can be drawn when $R_{1} s$ is $0 \%$, $Z_{\text {tip }}$ is $7.2 \%$, i $\beta_{1} s$ is $9^{\circ}$, and i $\left.\beta_{1}\right\lrcorner h$ is $5^{\circ}$. Figure 19 shows the three-dimensional geometry impeller of reference model and optimum model.

\section{Analyses of Numerical Analysis and Experiment Results}

The selected optimum model was verified by numerical analysis. Figure 20 shows total head and total efficiency curves according to the result of numerical analysis. Figure 20(a) shows total head curves of the reference and optimum models. The tendencies of total head curves from the reference and optimum models are same but total head curve of optimum model has higher value than that of reference model. Especially, the total head at design flow rate from the reference model is expected to be under $60 \mathrm{~m}$ not meeting the requirement of design specification. Optimum model was designed to have total head higher than $60 \mathrm{~m}$ which is design specification considering disk friction losses, mechanical losses, leakage losses, tip clearance effect, machine loss, roughness loss, and diffuser loss. Figure 20(b) shows the total 


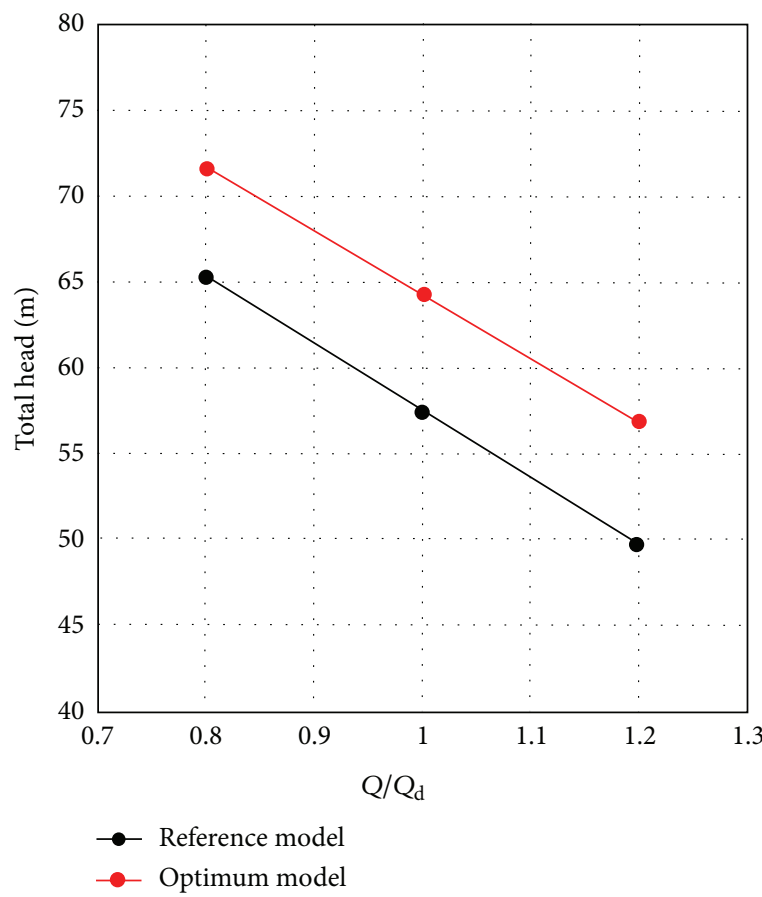

(a) Total head curve

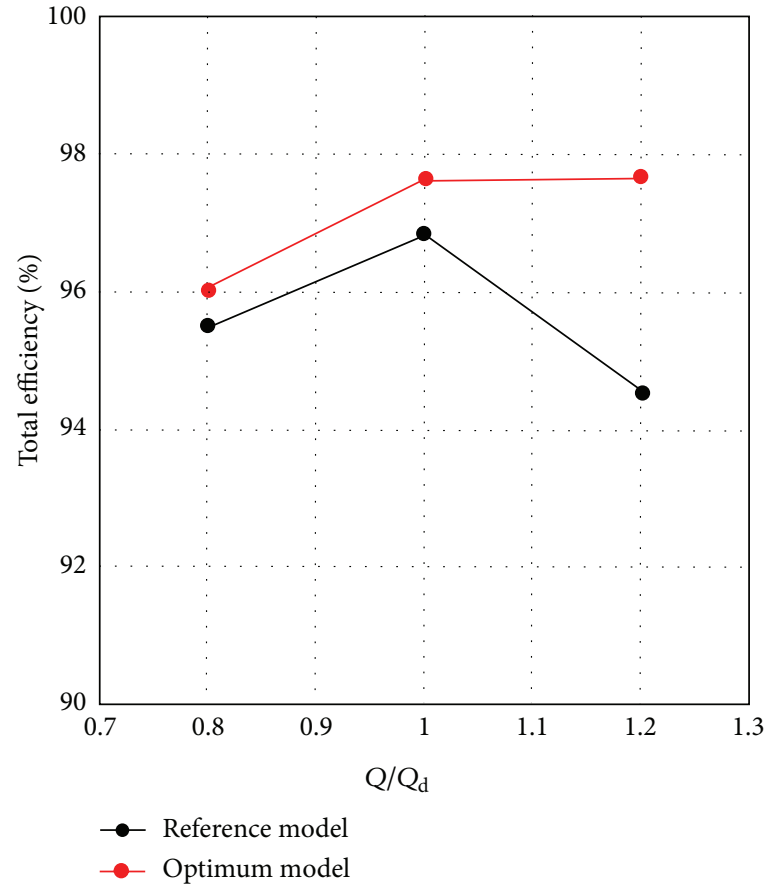

(b) Total efficiency curve

FIGURE 20: Comparison of total head and total efficiency curve.

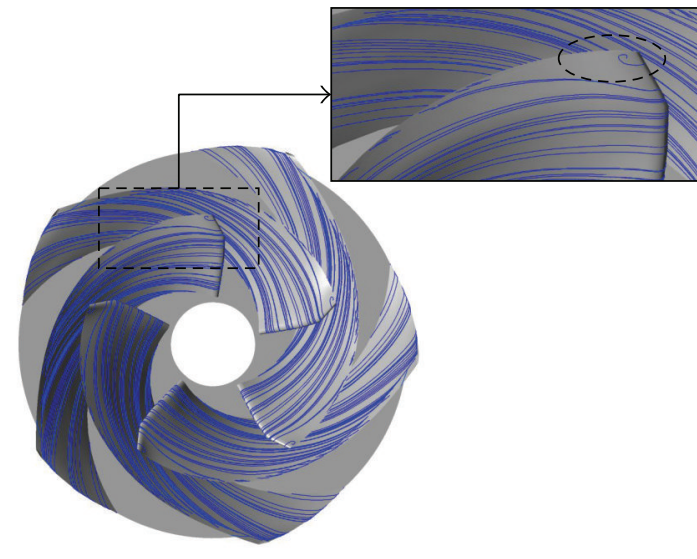

(a) Reference model

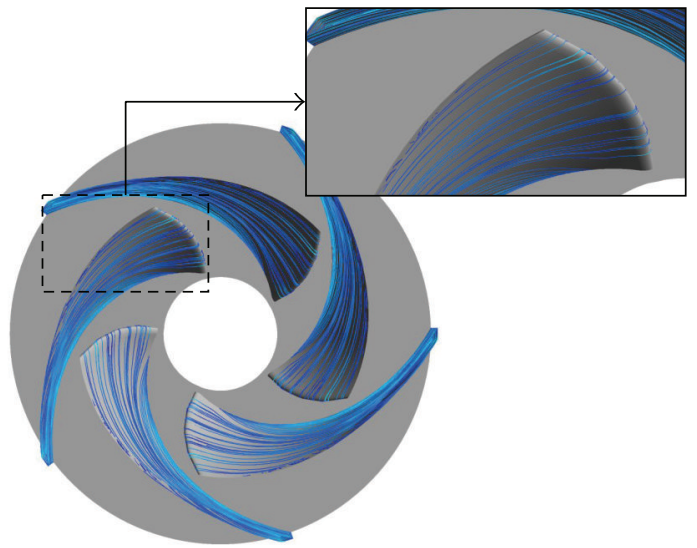

(b) Optimum model

FIgURE 21: Comparison of streamline at design flow rate.

efficiency curves of the reference and optimum models. In Figure 20(b), the total efficiency of optimum model is higher not only from the design flow rate but also from low and high flow rates than that of the reference model.

Figure 21 shows the comparison between reference and optimum models regarding streamline at impeller blades. In Figure 21(a), there is a section side with flow separation at inlet shroud of the reference model. The total efficiency seems to decrease as a result of this flow separation at impeller blades. In Figure 21(b), the streamline is stabilized at inlet shroud of the optimum model increasing total efficiency compared to reference model.
The NPSH curves for the reference and optimum models are shown in Figure 22. In Figure 22, the NPSHres for the reference and optimum models are $6.7 \mathrm{~m}$ and $5.7 \mathrm{~m}$, respectively. Consequently, the optimum model was improved as $1 \mathrm{~m}$ compared to the reference model. On the other hand, Figures 23 and 24 show the isosurfaces of vapor pressures of $-20 \mathrm{kPa}$ and $-40 \mathrm{kPa}$ at the NPSH $=8.0 \mathrm{~m}$ (design flow rate) and $\mathrm{NPSH}=5.9 \mathrm{~m}$, respectively. It seems that the quantity of vapor in both the models is similar as shown in Figure 23. In Figure 24, the dramatic decrease of the vapor in the optimum model is observed at the NPSH = $5.9 \mathrm{~m}$ in comparison with the reference model. It is thought 


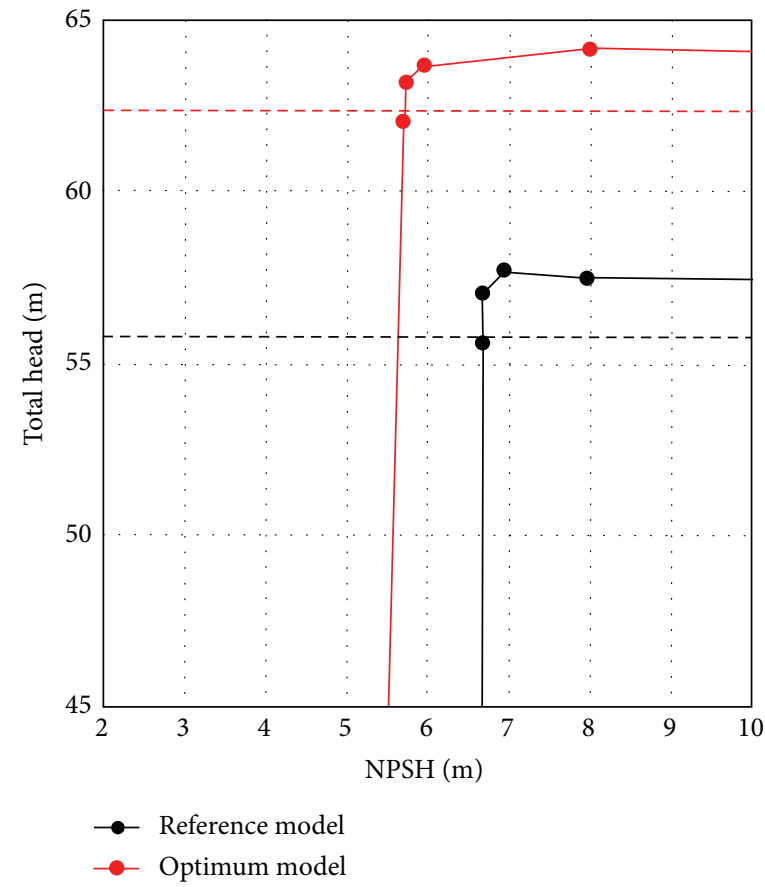

FIGURE 22: Comparison of NPSH curve at design flow rate.

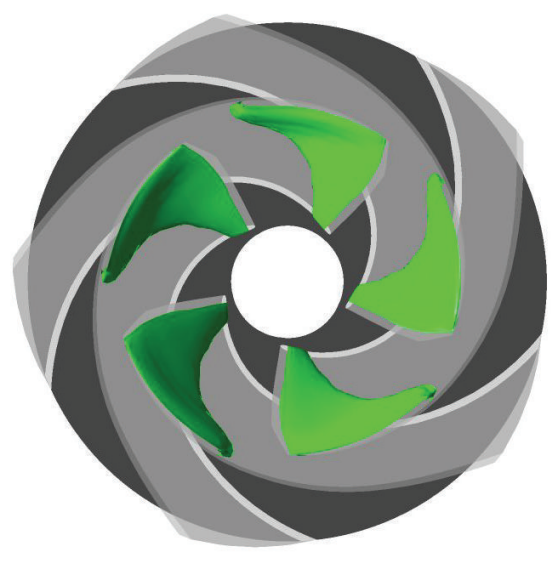

(a) Reference model

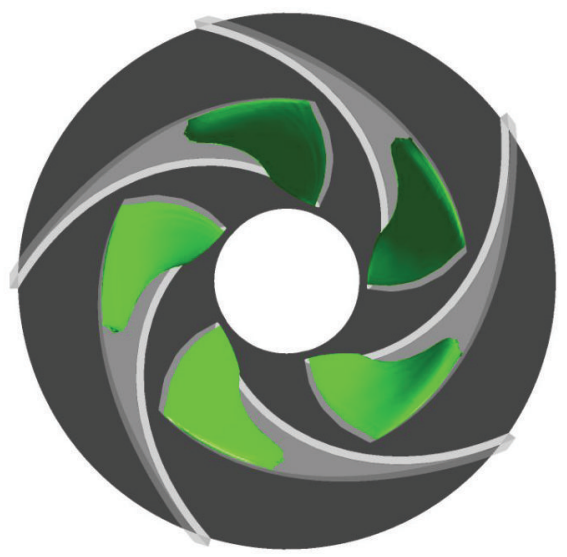

(b) Optimum model

FIGURE 23: Comparison of isosurface of vapor pressure at NPSH $=8.0 \mathrm{~m}$.

that the suction performance of the optimum model is improved by the considerable decrease of vapor in the suction side.

Figure 25 shows the NPSHre curves for the reference and optimum models. In Figure 25, it is apparent that the suction performance of the optimum model is improved in the entire flow rate, compared to the reference model. Table 4 shows the numerical results of the efficiency and NPSHre for the reference and optimum models at the design flow rate. According to the result of numerical analysis in Table 4, total head of optimum model is improved by the approximate $6 \mathrm{~m}$ than that of reference model. Moreover, it has shown $1 \mathrm{~m}$ and $0.81 \%$ improvements in the NPSHre and total efficiency, respectively, compared to the reference
TABLE 4: Comparison of total head, total efficiency, and NPSHre (CFD results).

\begin{tabular}{lccc}
\hline & $\begin{array}{c}\text { Total head } \\
(\mathrm{m})\end{array}$ & $\begin{array}{c}\text { Total } \\
\text { efficiency }(\%)\end{array}$ & NPSHre (m) \\
\hline Reference model & 57.48 & 96.84 & 6.7 \\
Optimum model & 64.25 & 97.65 & 5.7 \\
\hline
\end{tabular}

model. Therefore, the optimum design produced mostly stable flows in the impeller passage.

The overall experimental apparatus used in the performance test is illustrated in Figure 26, which shows the installation of the test pump [20]. The test pump is connected 


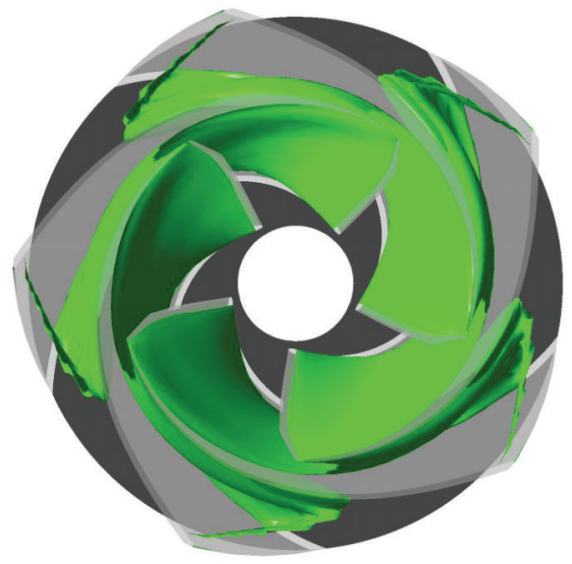

(a) Reference model

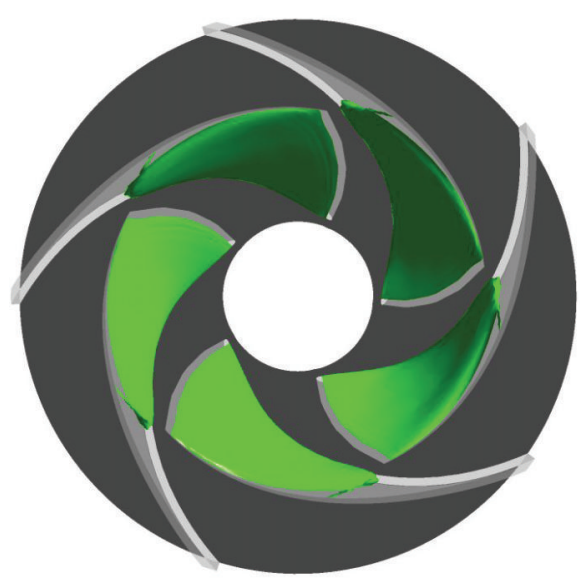

(b) Optimum model

FIGURE 24: Comparison of isosurface of vapor pressure at $\mathrm{NPSH}=5.9 \mathrm{~m}$.

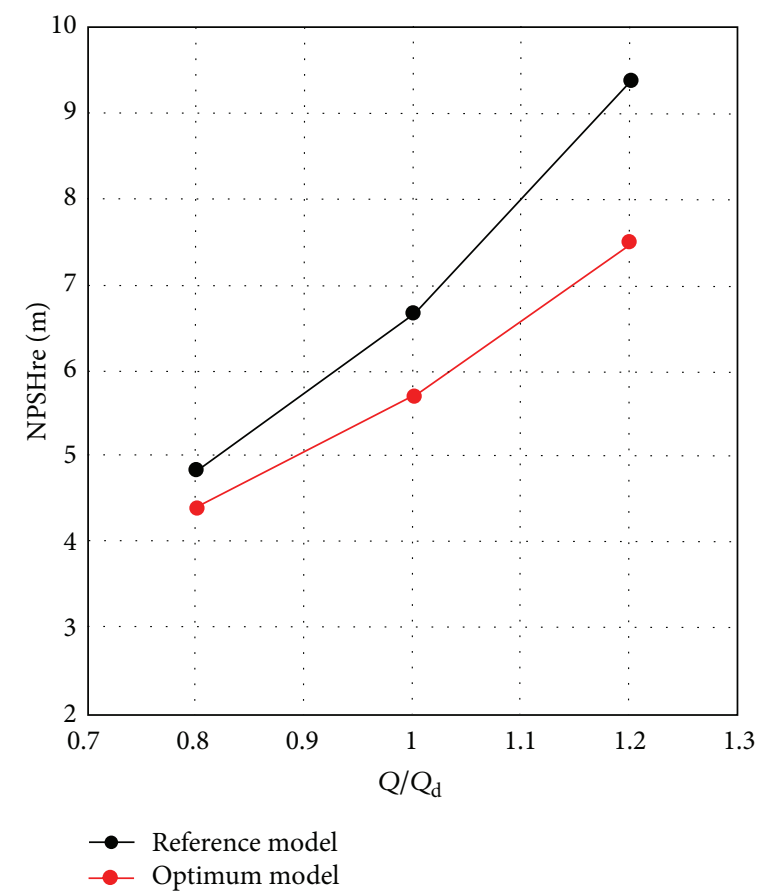

Figure 25: Comparison of NPSHre curve.

to the electric motor which is controlled by an inverter and a data acquisition system. As shown in Figure 26, the main component devices for the experimental apparatus consist of the flow meter, control valve, heat exchanger, booster pump, thermometer, damping valve, and pressure gauge. The measuring instruments and their uncertainties used for the performance test are tabulated in Table 5 .

The numerical and experimental results for the total head, total efficiency, and NPSHre of the optimum model are compared in Figure 27. As shown in Figures 27(a) and 27(b), although the numerical and experimental results are different in terms of quantitative value, they are similar in tendency.
TABLE 5: Specifications of a measurement device.

\begin{tabular}{lc}
\hline Measurement device & Uncertainty (\%) \\
\hline Torque meter & \pm 2 \\
Flow meter & \pm 2 \\
Rotational sensor & \pm 0.3 \\
Absolute pressure transducer & \pm 2 \\
Differential pressure transducer & \pm 2 \\
Thermocouple & \pm 2 \\
\hline
\end{tabular}

In Figure 27(c), they are also similar in tendencies in the NPSHre curves but show the bigger difference at especially 


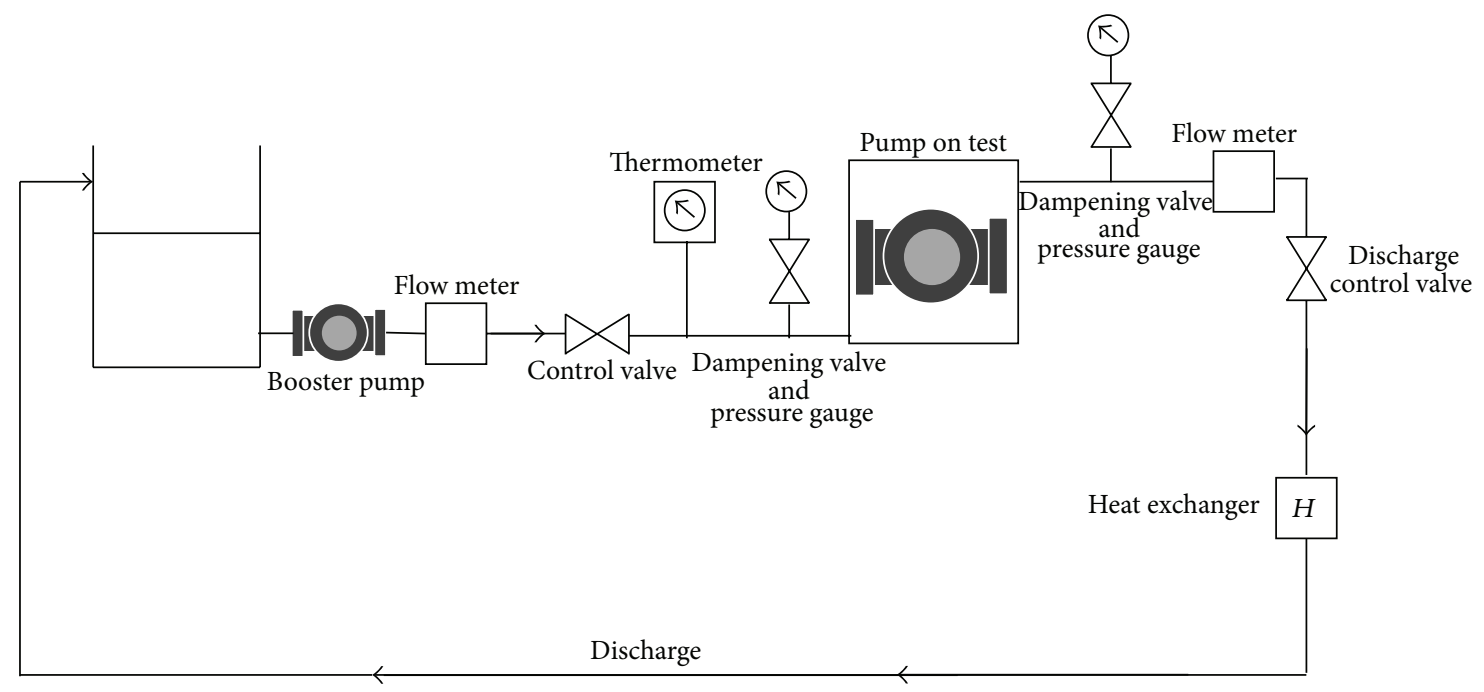

FIgURE 26: Schematic diagram of experimental apparatus for a mixed-flow pump [20].

the high flow rate. This is probably explained by the fact that bell mouth of inlet part, disk friction losses, mechanical losses, leakage losses, tip clearance effect, machine loss, roughness loss, and diffuser loss were not considered for the numerical analysis. First of all, the main effect on a relatively large error between the CFD and experiment results is due to no consideration of the tip clearance. These results illustrate the enhancement of the pump impeller's performances as a result of the optimization.

\section{Conclusion}

A numerical optimization on the improvement of suction performance and hydraulic efficiency of a mixed-flow pump impeller was conducted in this work. The main conclusions of this work are summarized as follows.

(1) In this work, the impeller's meridional plane and vane plane development were employed as the design variables to improve the suction performance and total efficiency of the mixed-flow pump. According to the result of $2^{k}$ factorial, it showed that the suction performance and total efficiency had an opposite trend with the selected design variables.

(2) In the analyses of the main effects plot and Pareto chart, the $\mathrm{i} \beta_{1} s$ was more sensitive than other variables in terms of the NPSHre and total efficiency. On the other hand, the $R_{1} s$ affected considerably to total efficiency, whereas it did not affected absolutely to the NPSHre.

(3) As a result of the optimization, the total head of the optimum model was numerically improved by the approximate $6 \mathrm{~m}$, and moreover its numerical result showed $1 \mathrm{~m}$ and $0.81 \%$ improvements in the NPSHre and total efficiency, respectively, in comparison with the reference model.
(4) The numerical and experimental results for the total head, total efficiency, and NPSHre of the optimum model were compared. Although the numerical and experimental results for the total head, total efficiency, and NPSHre were different in terms of quantitative value, they were similar in tendency. Therefore, the numerical optimization method based on the impeller's meridional plane and vane plane development can be effectively used for improving hydraulic performance of the mixed-flow pump.

\section{Nomenclature}

CFD: Computational fluid dynamics

DOE: Design of experiments

h: Hub

$H_{\mathrm{d}}$ : Designed total head (m)

$H_{\mathrm{t}}$ : Total head (m)

$M: \quad$ Meridional length $(\mathrm{m})$

$N$ : $\quad$ Rotational speed ( $\mathrm{r} / \mathrm{min})$

NPSH: Net positive suction head (m)

NPSHre: Net positive suction head required $(\mathrm{m})$

$N_{\mathrm{s}}$ : $\quad$ Specific speed $\left(\mathrm{r} / \mathrm{min}, \mathrm{m}^{3} / \mathrm{min}, \mathrm{m}\right)$

$P_{\text {in: }}$ Inlet pressure (pa)

$P_{\mathrm{v}}: \quad$ Vapor pressure $(\mathrm{pa})$

Q: $\quad$ Flow rate $\left(\mathrm{m}^{3} / \mathrm{hr}\right)$

$Q_{\mathrm{d}}: \quad$ Design flow rate $\left(\mathrm{m}^{3} / \mathrm{hr}\right)$

$R_{1}$ : $\quad$ Radius of inlet part $(\mathrm{m})$

$R_{2}$ : $\quad$ Radius of outlet part (m)

$s: \quad$ Shroud

$Z_{\text {tip }}$ : Axial direction from inlet/outlet parts of the shroud (m)

$\Phi_{1}$ : $\quad$ Inclined angle of the leading edge $\left({ }^{\circ}\right)$

$\Phi_{2}$ : $\quad$ Inclined angle of the trailing edge $\left({ }^{\circ}\right)$ 


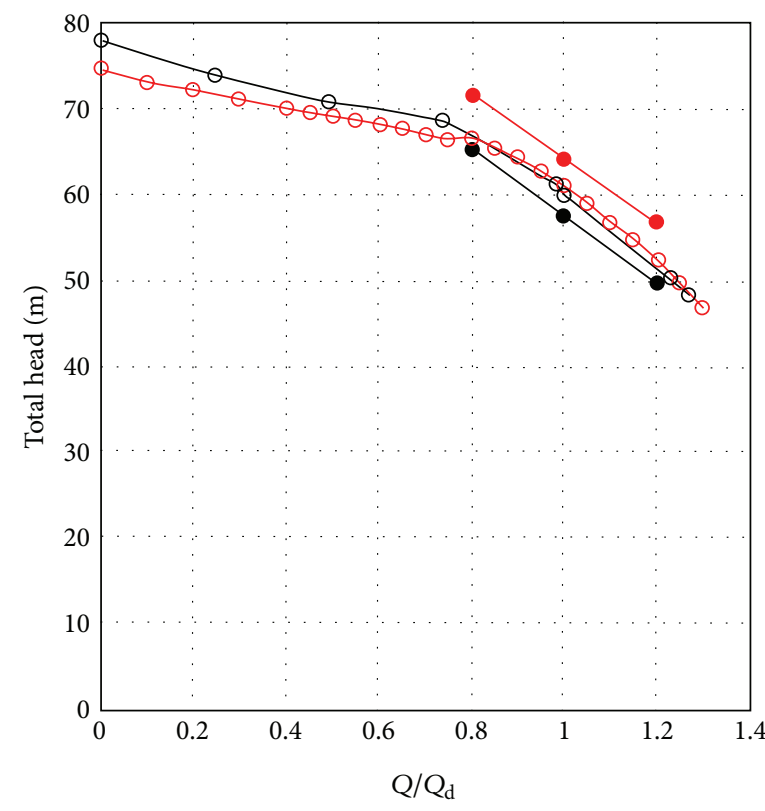

Reference model_CFD $\multimap$ Reference model_Exp Optimum model_CFD -0 Optimum model_Exp

(a) Performance evaluation of the total head

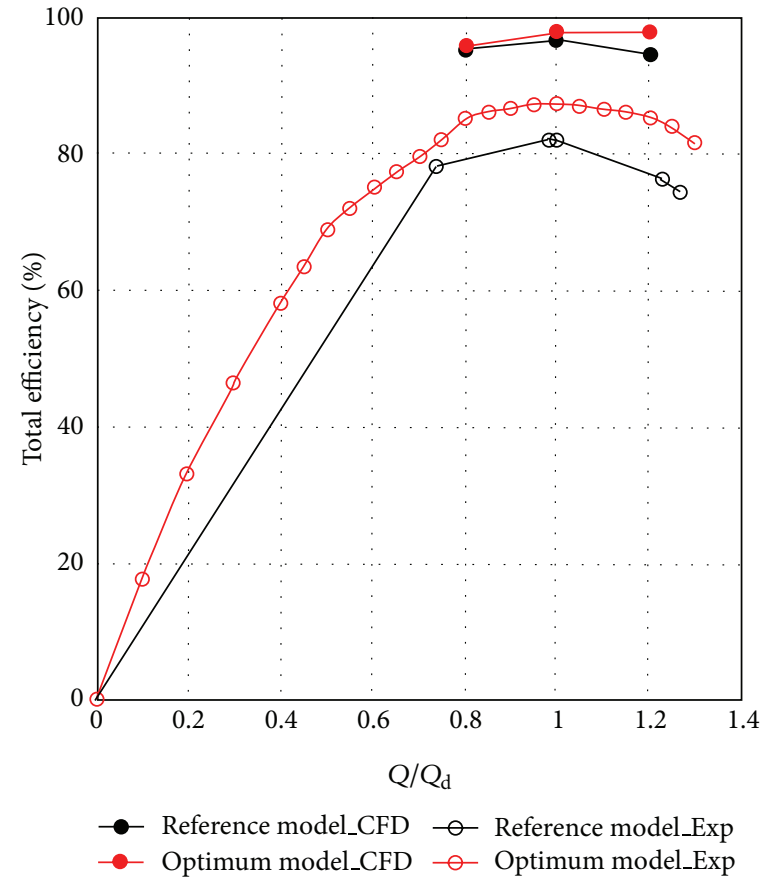

(b) Performance evaluation of the total efficiency

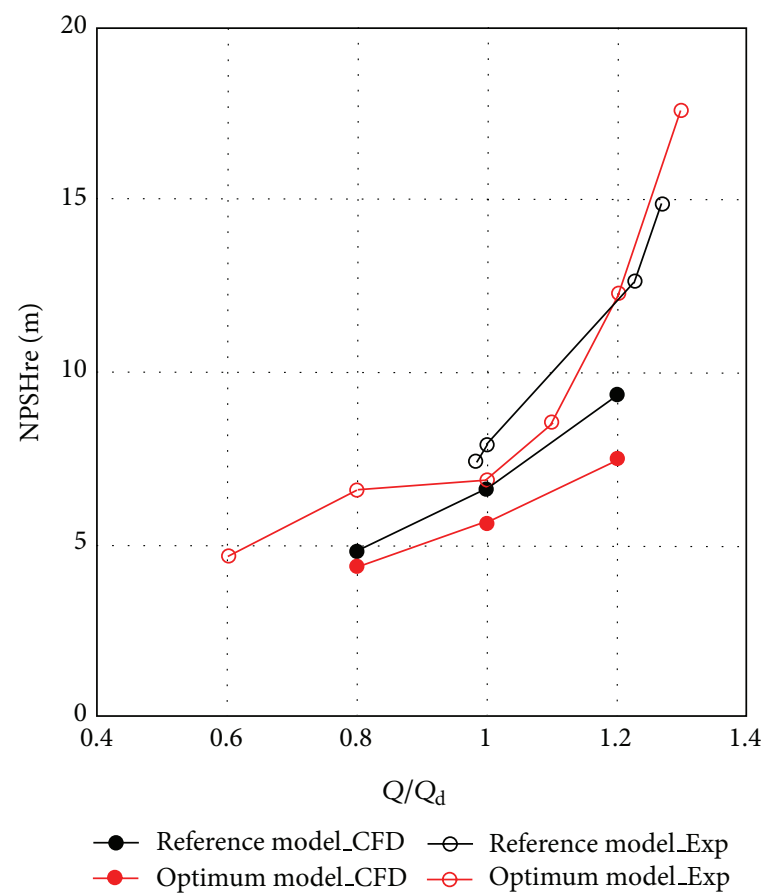

(c) Performance evaluation of the NPSHre

FIGURE 27: Comparative analysis of the performance evaluation (experimental versus numerical analysis).

$\beta_{1}$ : Inlet angle of the impeller $\left(^{\circ}\right)$

$\mathrm{i} \beta_{1}$ : Incidence angle $\left({ }^{\circ}\right.$ )

$\theta_{1}$ : Inlet angle from meridional curve $\left({ }^{\circ}\right)$

$\theta_{2}$ : Outlet angle from meridional curve $\left(^{\circ}\right)$

$\% L$ : Straight line from outlet part (\%)
$\% \mathrm{CP}_{1}$ : Control points of inlet part (\%)

$\% \mathrm{CP}_{2}$ : Control points of outlet part $(\%)$

$\% \beta_{1}$ : Portion of same inlet blade angle (\%)

$\% \beta_{2}$ : Portion of same outlet blade angle (\%)

$\omega: \quad$ Angular velocity $(\mathrm{m} / \mathrm{s})$. 


\section{Conflict of Interests}

The authors declare that there is no conflict of interests regarding the publication of this paper

\section{Acknowledgments}

This research was supported by a grant (no. 10044860) from the Korea Institute of Industrial Technology Evaluation and Planning (ITEP) that is funded by the Ministry of Science, ICT and Future Planning.

\section{References}

[1] N. Nariman-Zadeh, N. Amanifard, A. Hajiloo, P. Ghalandari, and B. Hoseinpoor, "Multi-objective pareto optimization of centrifugal pump using genetic algorithms," in Proceedings of the 11th WSEAS International Conference on Computers, pp. 135139, Crete Island, Greece, 2007.

[2] R. K. Ursem, "Centrifugal pump design: three benchmark problems for many-objective optimization," Grundfos Management Technical Report 2010-01, 2010.

[3] J. Kurokawa, K. Matsumoto, J. Matsui, and T. Kitahora, "Performances of centrifugal pumps of very low specific speed," in Proceedings of the 19th IAHR Symposium on Hydraulic Machinery and Cavitation, pp. 833-842, Singapore, 1998.

[4] J. Kurokawa, K. Matsumoto, J. Matsui, and H. Imamura, "Development of high efficiency volute pump of very low specific speed," in Proceedings of the 6th Asian International Conference on Fluid Machinery, pp. 250-255, Johor, Malaysia, 2000.

[5] J. F. Gulich, Centrifugal Pumps Second Edition, Springer, Heidelberg, Germany, 2010.

[6] L. Bachus and A. Custodio, Know and Understand Centrifugal Pumps, Elsevier, New York, NY, USA, 2006.

[7] E. Grist, Cavitation and the Centrifugal Pump: A Guide for Pump Users, Taylor \& Francis, 1998.

[8] A. Goto and M. Zangeneh, "Hydrodynamic design of pump diffuser using inverse design method and CFD," Journal of Fluids Engineering, vol. 124, no. 2, pp. 319-328, 2002.

[9] H. Bing and S. Cao, "Optimal design of mixed-flow pump impeller based on direct inverse problem iteration and genetic algorithm," in Proceedings of the ASME-JSME-KSME Joint Fluids Engineering Conference (AJK '11), vol. 8, pp. 803-810, Shizuoka, Japan, July 2011.

[10] J. H. Kim and K. Y. Kim, "Optimization vane diffuser in a mixed-flow pump for high efficiency design for high efficiency design," International Journal of Fluid Machinery and Systems, vol. 4, no. 1, pp. 172-178, 2011.

[11] J. Stepanoff, Centrifugal and Axial Flow Pumps, John Wiley \& Sons, New York, NY, USA, 1957.

[12] B. Neumann, "The interaction between geometry and performance of a centrifugal pump," in Mechanical Engineering, pp. 74-87, Publications Limited, London, UK, 1991.

[13] S. Kim, Y. S. Choi, K. Y. Lee, and J. Y. Yoon, "Design optimization of centrifugal pump impellers in a fixed meridional geometry using DOE," International Journal of Fluid Machinery and Systems, vol. 2, no. 2, pp. 172-178, 2009.

[14] S. Kim, Y. S. Choi, K. Y. Lee, and J. H. Kim, "Design optimization of mixed-flow pump in a fixed meridional shape," International Journal of Fluid Machinery and Systems, vol. 4, no. 1, pp. 14-24, 2011.
[15] Help Navigator, ANSYS CFX, Release 11.0, ANSYS CFX-Solver Theory Guide.

[16] J.-H. Kim, J.-W. Kim, and K.-Y. Kim, "Axial-flow ventilation fan design through multi-objective optimization to enhance aerodynamic performance," ASME Journal of Fluids Engineering, vol. 133, no. 10, Article ID 101101, 2011.

[17] F. R. Menter, "Zonal two equation $\kappa$ - $\omega$ turbulence models for aerodynamic flows," in Proceedings of the AIAA 24th Fluid Dynamics Conference, pp. 789-792, 1993.

[18] Help Navigator, "ANSYS CFX, Release 11.0, ANSYS CFX-Solver Modeling Guide, Multiphase Flow Modeling, Interphase Mass Transfer, Cavitation Model".

[19] Help Navigator, ANSYS CFX, Release 11.0, ANSYS CFX-Solver Theory Guide, Turbulence and Wall Function Theory, Eddy Viscosity Turbulence Models, Two Equation Turbulence Models.

[20] ANSI/HI 1.6, American National Standard for Centrifugal Pump Tests, Hydraulic Institute, Parsippany, NJ, USA, 2000.

[21] U. H. Jung, Y. S. Choi, and K. Y. Lee, "Optimum design of volute configuration in a sirocco fan using CFD and DOE," International Journal of Air-Conditioning and Refrigeration, vol. 17, no. 2, pp. 68-73, 2007.

[22] R. H. Myers and D. C. Montgomery, Response Surface Methodology: Process and Process and Product Optimization Using Designed Experiments, A Wiley-Interscience Publication, New York, NY, USA, 2nd edition, 2002.

[23] C. R. Hick and K. V. Turner, Fundamental Concepts in the Design of Experiments, Oxford University Press, New York, NY, USA, 5th edition, 1999.

[24] V. S. Lobanoff and R. R. Ross, Centrifugal Pumps Design \& Application, Gulf Publishing, London, UK, 2nd edition, 1992.

[25] T. Wright, Fluid Machinery Performance, Analys, and Design, CRC Press, New York, NY, USA, 1999.

[26] M. Volk, Pump Characteristics and Applications, Taylor \& Francis Group, Columbus, Ohio, USA, 2nd edition, 2005. 


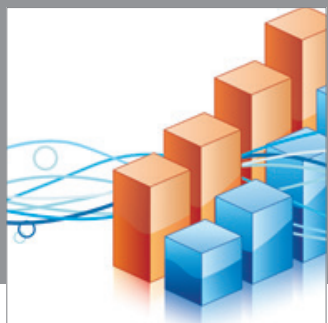

Advances in

Operations Research

mansans

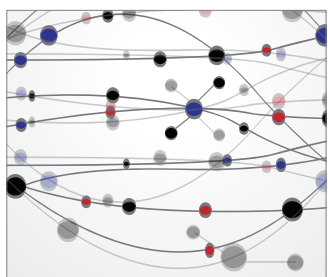

The Scientific World Journal
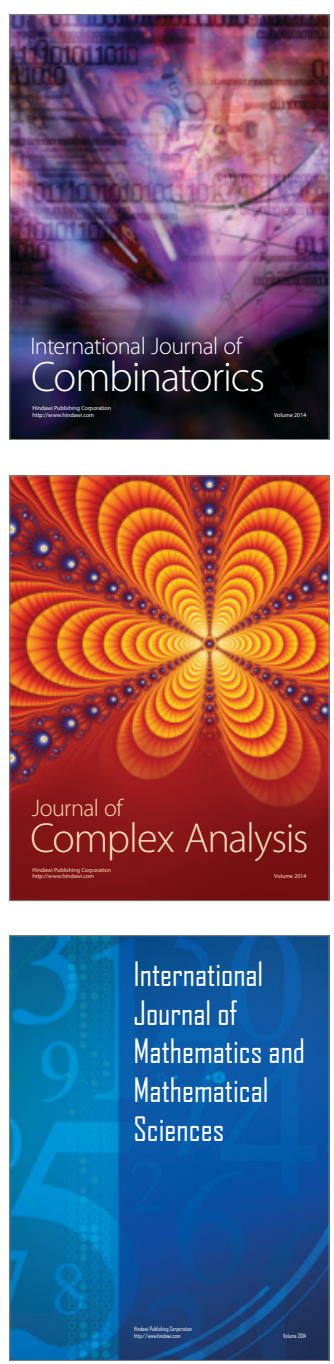
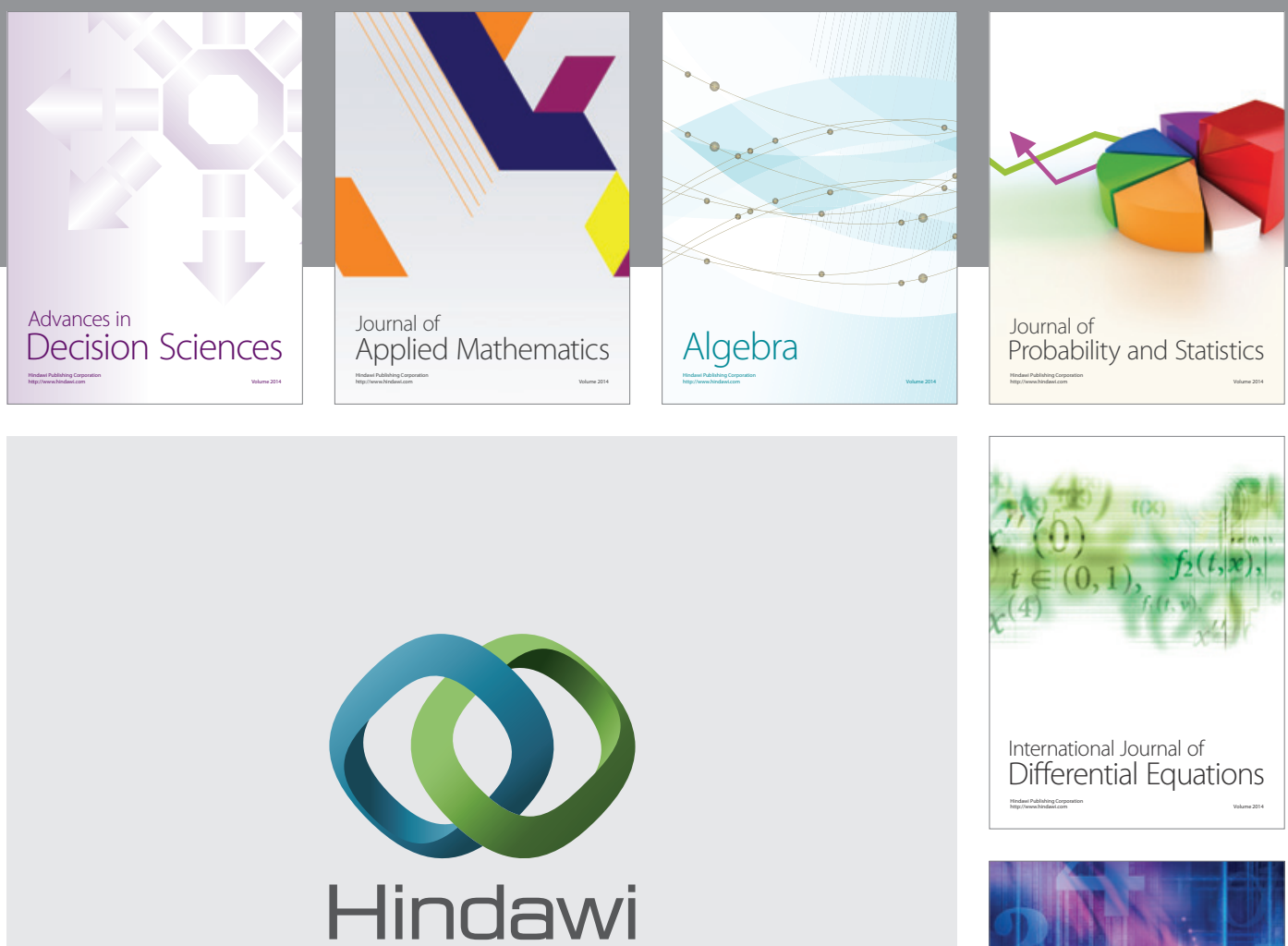

Submit your manuscripts at http://www.hindawi.com
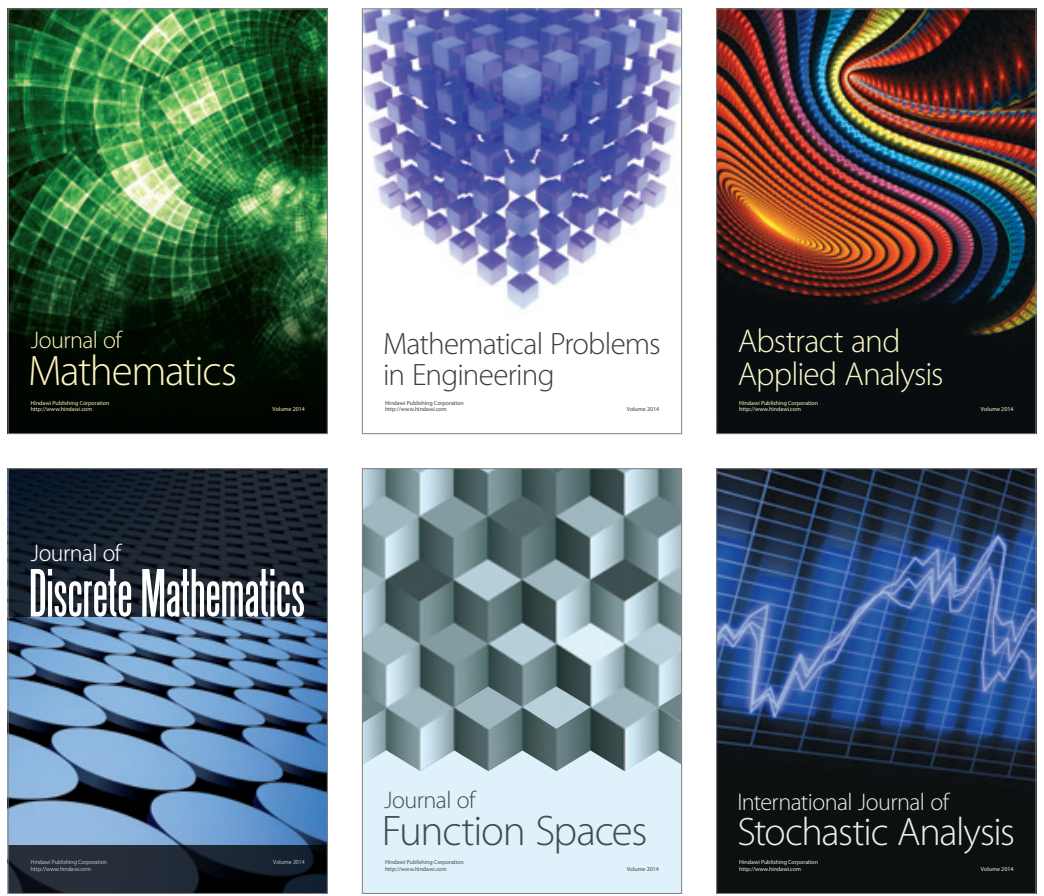

Journal of

Function Spaces

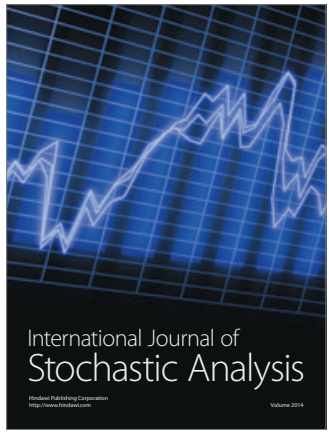

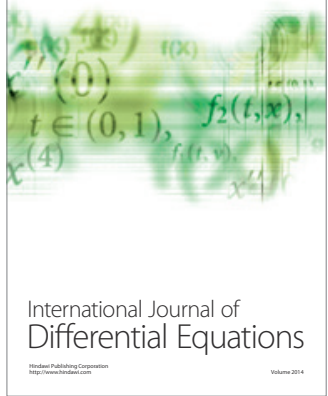
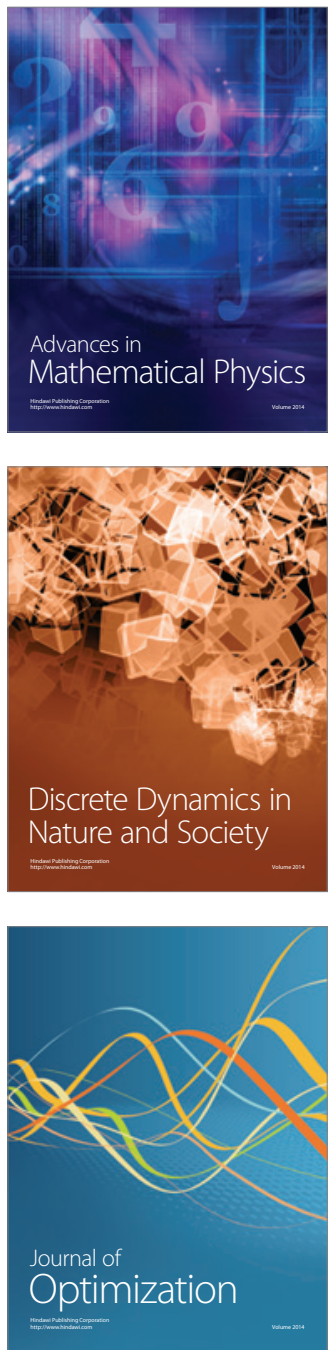